\title{
Polygons pulled from an adsorbing surface
}

\author{
A J Guttmann*, E J Janse van Rensburg ${ }^{\dagger}$, I Jensen* and S G \\ Whittington $\ddagger$ \\ *School of Mathematics and Statistics, The University of Melbourne, Parkville, Victoria 3010, \\ Australia \\ ${ }^{\dagger}$ Department of Mathematics \& Statistics, York University, M3J 1P3, Toronto, Canada \\ ${ }^{\ddagger}$ Department of Chemistry, University of Toronto, M5S 3H6, Toronto, Canada
}

6 September 2021

\begin{abstract}
We consider self-avoiding lattice polygons, in the hypercubic lattice, as a model of a ring polymer adsorbed at a surface and either being desorbed by the action of a force, or pushed towards the surface. We show that, when there is no interaction with the surface, then the response of the polygon to the applied force is identical (in the thermodynamic limit) for two ways in which we apply the force. When the polygon is attracted to the surface then, when the dimension is at least 3 , we have a complete characterization of the critical force-temperature curve in terms of the behaviour, (a) when there is no force, and, (b) when there is no surface interaction. For the 2-dimensional case we have upper and lower bounds on the free energy. We use both Monte Carlo and exact enumeration and series analysis methods to investigate the form of the phase diagram in two dimensions. We find evidence for the existence of a mixed phase where the free energy depends on the strength of the interaction with the adsorbing line and on the applied force.
\end{abstract}

PACS numbers: 82.35.Lr,82.35.Gh,61.25.Hq

AMS classification scheme numbers: 82B41, 82B80, 65C05

Submitted to: J Phys A 


\section{Introduction}

The theory of polymer adsorption at an impenetrable surface is a well established subject. Useful reviews can be found in [7] and [26]. One of the standard models is self-avoiding walks, or SAWs, confined to a half-space and interacting with the confining line or plane. For this problem we have some rigorous results $[16,24]$ that establish the existence of a phase transition and provide useful information about the behaviour of the free energy as the temperature is varied. More detailed information comes from a variety of numerical investigations that, among other things, give quite precise information about the location of the phase transition $[3,11,13,20,29]$ and strongly suggest that the transition is second order [11, 20, 29].

With the advent of atomic force microscopy [19, 45] it has become possible to pull an adsorbed polymer off a surface at which it is adsorbed. In principle it is possible to measure the temperature dependence of the critical force for desorption and the stress-strain curves. It is only quite recently that the effects of a force have been investigated for the self-avoiding walk model $[1,13,28,30,36,37,41]$.

Consider the $d$-dimensional hypercubic lattice, $\mathbb{Z}^{d}$, and attach a coordinate system $\left(x_{1}, x_{2}, \ldots x_{d}\right)$ so that each vertex of the lattice has integer coordinates. Suppose that $c_{n}^{+}$is the number of $n$-edge self-avoiding walks that start at $(0,0, \ldots 0)$ and have all vertices in the half-space $x_{d} \geq 0$. It is known [44] that

$$
\lim _{n \rightarrow \infty} n^{-1} \log c_{n}^{+}=\kappa_{d}
$$

where $\kappa_{d}$ is the connective constant of the lattice [15]. Each vertex of the walk in the hyperplane $x_{d}=0$ is called a visit. Let $c_{n}^{+}(v, h)$ be the number of these walks with $v+1$ visits and having the $x_{d}$-coordinate of their last vertex equal to $h$, which we call the height of the last vertex. Define the partition function

$$
C_{n}^{+}(a, y)=\sum_{v, h} c_{n}^{+}(v, h) a^{v} y^{h} .
$$

It is known [30] that the limit

$$
\lim _{n \rightarrow \infty} n^{-1} \log C_{n}^{+}(a, y)=\psi(a, y)
$$

exists. $\psi(a, y)$ is the (reduced, limiting) free energy.

We can interpret the two fugacities $a$ and $y$ as

$$
a=\exp \left(-\epsilon / k_{B} T\right) \quad \text { and } \quad y=\exp \left(f / k_{B} T\right)
$$

where $k_{B}$ is Boltzmann's constant, $T$ is the absolute temperature, $\epsilon$ is the energy associated with a vertex in the surface and $f$ is the force applied at the last vertex, normal to the surface. For adsorption to occur $\epsilon<0$ so that the interaction with the surface is attractive. For the walk to be desorbed by the action of the force, $f>0$.

If we set $f=0$, so that $y=1$, we have the pure adsorption problem. Write $\psi(a, 1)=\kappa(a)$, the free energy for pure adsorption. We know $[16,24]$ that there is a critical value of $a, a_{c}>1$, such that $\kappa(a)=\kappa(1)=\kappa_{d}$ for $a \leq a_{c}$ and $\kappa(a)>\kappa(1)$ for $a>a_{c}$ so that the free energy is singular at $a=a_{c}$, corresponding to the adsorption transition. Similarly if we set $\epsilon=0$ so that $a=1$ we have no (attractive) interaction with the surface and there is no adsorbed phase. The free energy is $\lambda(y)=\psi(1, y)$ and we know that $\lambda(y)$ is singular at $y=y_{c}=1[1]$. There is a transition from a free phase to a ballistic phase at $y=1$ [1], see also [22, 23]. 


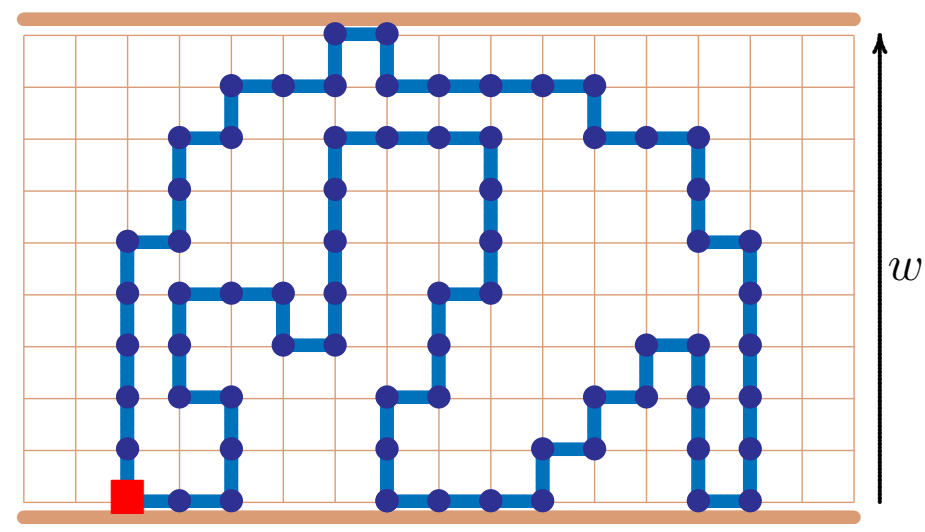

Figure 1. A polygon in a slab of width $w$. If $w=\infty$ the polygon is in a half-space.

Returning to the full problem, we know [30] that for $a \geq a_{c}$ and $y \geq 1$

$$
\psi(a, y)=\max [\kappa(a), \lambda(y)] .
$$

This gives a complete characterization of the phase boundary between the adsorbed and ballistic phases in terms of the behaviour when $\epsilon=0$ and when $f=0$. This was used in [13] to give very precise numerical estimates of the location of the phase boundary when $d=2$. Numerical estimates using a different approach are given in [37] for both $d=2$ and $d=3$. In addition we know [13] that the phase transition from the adsorbed to the ballistic phase is first order.

These results raise a variety of new questions. For the self-avoiding walk model, what happens if the force is applied somewhere other than at the last vertex? What happens if the force is applied at an angle to the surface? Not all polymers are linear and there are interesting questions about the behaviour of ring polymers or branched polymers when they are pulled off a surface at which they are adsorbed. That is, how does the architecture of the polymer affect its behaviour?

Does it matter where the force is applied? If the force is applied at the top vertex, i.e. at the vertex furthest from the surface, this is equivalent to confining the walk (or polygon, etc.) between two parallel lines or planes and requiring at least one vertex in each plane, then applying a force to move the confining plane. For the walk problem we know that the limiting free energy is the same when the force is applied in this way or at the terminal vertex [31, 32]. If the force is applied at an interior vertex, the free energy depends on which vertex the force is applied to, and in some circumstances, an additional phase can be present [33]. Some results for staircase polygons [2] suggest a phase diagram with a mixed adsorbed and ballistic phase.

In this paper we begin to investigate the issue of polymer architecture. We consider a ring polymer adsorbed at a surface, being pulled off the surface by a force applied in a particular way.

\section{Definition of the model and statement of results}

A standard model of ring polymers is self-avoiding lattice polygons or polygons, or SAPs, for short. These are embeddings of the circle graph in a lattice. Each vertex has degree 2 . Let $p_{n}$ be the number of (undirected, unrooted) $n$-edge polygons in $\mathbb{Z}^{d}$, counted modulo translation. In two dimensions, $p_{4}=1, p_{6}=2, p_{8}=7$, etc. Clearly $p_{2 m+1}=0$. Let $p_{n}(v, s)$ be the number of $n$-edge 


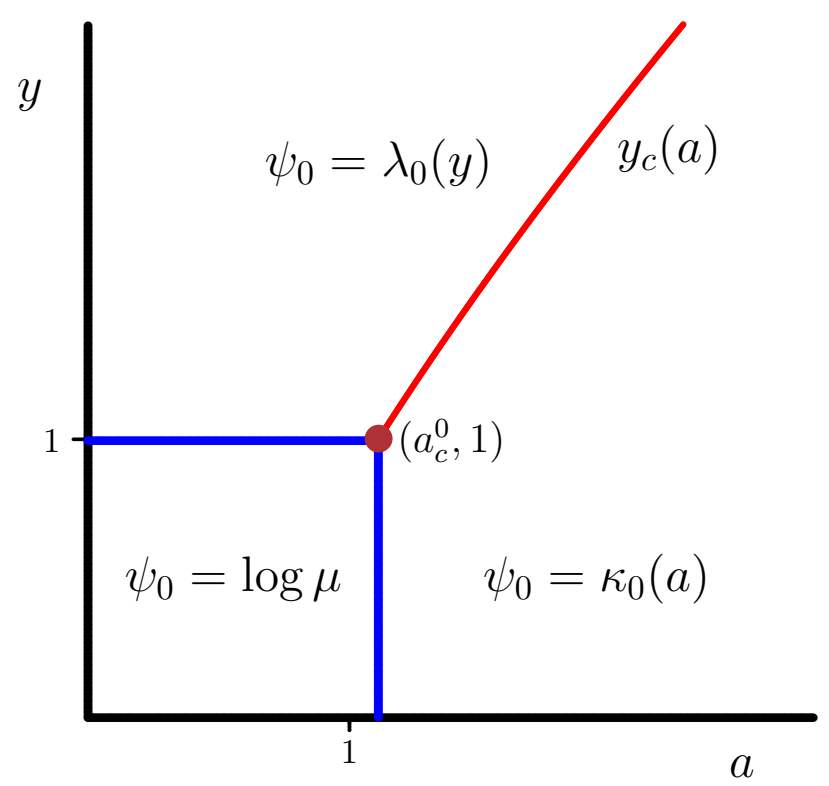

Figure 2. The phase diagram of pulled adsorbing polygons in $d \geq 3$ dimensions. For $y \leq 1$ and $a \leq a_{c}^{0}$ the free energy is $\psi_{0}=\log \mu$. This corresponds to a free phase with phase boundaries separating it from the ballistic phase at $y=1$ and from the adsorbed phase. In the ballistic phase the free energy is $\psi_{0}=\lambda_{0}(y)=\lambda(\sqrt{y})$. In the adsorbed phase the free energy is $\psi_{0}=\kappa_{0}(a)=\kappa(a)$. The solution of $\lambda_{0}(y)=\kappa_{0}(a)$ is a critical curve $y_{c}(a)$ separating the ballistic and adsorbed phases. This phase boundary is first order.

polygons with all vertices having $x_{d} \geq 0, v+2$ visits $(v \geq 0)$ and span in the $x_{d}$-direction equal to $s$, counted modulo translation parallel to the surface.

Define the partition function

$$
P_{n}(a, y)=\sum_{v \geq 0, s \geq 0} p_{n}(v, s) a^{v} y^{s} .
$$

The fugacities are again interpreted as in equation (1.4) and now the force is applied at the highest vertex.

In this paper we show that in $d \geq 3$ dimensions this model has a thermodynamic limit with free energy defined by

$$
\psi_{0}(a, y)=\lim _{n \rightarrow \infty} \frac{1}{n} \log P_{n}(a, y) .
$$

In addition, we show that, in this case, for $a \geq a_{c}^{0}$ (where $a_{c}^{0}$ is the critical adsorption fugacity for adsorbing polygons) and $y \geq 1$,

$$
\psi_{0}(a, y)=\max \left[\kappa_{0}(a), \lambda_{0}(y)\right]
$$

where $\kappa_{0}(a)$ is the free energy of adsorbing polygons in the absence of a pulling force, and $\lambda_{0}(y)$ is the free energy of a pulled polygon in the absence of an adsorption fugacity. The resulting phase diagram of this model is sketched in Figure 2. We know that the phase boundary between the free phase and the adsorbed phase is a vertical line, and the phase boundary between the free phase and the ballistic phase is a horizontal line.

In $d=2$ dimensions, our results are less complete. 


\section{Polygons adsorbing at a surface}

If we consider polygons adsorbing at a surface with no applied force $(y=1)$ we have the following theorem:

Theorem 1 (Soteros) The thermodynamic limit

$$
\lim _{n \rightarrow \infty} n^{-1} \log P_{n}(a, 1) \equiv \kappa_{0}(a)<\infty
$$

exists. Moreover, $\kappa_{0}(a)$ is a convex function of $\log a$ and hence is continuous and differentiable almost everywhere.

The proof of this theorem follows from results in [42], in Sections 3 and 4.

When $d \geq 3$ it is relatively straightforward to show that $\kappa_{0}(a)=\kappa(a)$ so the location of the adsorption transition is the same as that of walks. See Section 3 of [42] for a proof when $d=3$ that can be extended to $d>3$ without much difficulty. When $d=2$ the situation is slightly different because a polygon cannot lie entirely in the confining line. We have the following theorem.

Theorem 2 (Soteros) Suppose that $d=2$. When $a \leq 1$ then $\kappa_{0}(a)=\kappa_{0}(1)=\kappa_{2}$. When $a>1$

$$
\max \left[\kappa_{2},(1 / 2) \log a\right] \leq \kappa_{0}(a) \leq \kappa_{2}+(1 / 2) \log a .
$$

A proof is given in Section 4 of [42].

This theorem implies that there is an adsorption transition at $a=a_{c}^{0}$ where

$$
1 \leq a_{c}^{0} \leq \exp \left[2 \kappa_{2}\right]
$$

and, with a little more effort, the upper bound can be made strict. By deleting a suitable edge each polygon can be converted into a terminally attached self-avoiding walk so $\kappa_{0}(a) \leq \kappa(a)$ and this implies that $a_{c}^{0} \geq a_{c}$. Since we know [16] that $a_{c}>1$ this implies that $a_{c}^{0}>1$ so both inequalities in (3.3) are strict. It is an open question as to whether the two critical points are identical or not.

\section{Polygons pulled from a non-interacting surface}

In this section we consider a polygon attached to an impenetrable surface and pulled away from the surface. There is no attractive interaction so $a=1$. The force is conjugate to the span $(s)$ of the polygon in the $x_{d}$-direction and the partition function is

$$
P_{n}(1, y)=\sum_{v \geq 0, s \geq 0} p_{n}(v, s) y^{s} .
$$

The existence of the limit

$$
\lim _{n \rightarrow \infty} n^{-1} \log P_{n}(1, y)=\lambda_{0}(y)
$$

is established in [27]. In addition we know [27] that $\lambda_{0}(y)$ is a convex function of $\log y$ (and hence continuous) and, for $y \geq 1, \lambda_{0}(y)$ satisfies the bounds

$$
\max \left[\lambda_{0}(1),(1 / 2) \log y\right] \leq \lambda_{0}(y) \leq \lambda_{0}(1)+(1 / 2) \log y .
$$

Note that $\lambda_{0}(1)=\kappa_{d}$. 


\subsection{Polygons in a slit or slab}

It will be useful to consider polygons with at least one vertex in the bottom boundary of a slit or slab of width $w$ in the hypercubic lattice. Let $\pi_{n}(w)$ be the number of polygons of length $n$ with highest vertices at height $w$, so that they fit in a slab or slit of width $w$ (see Figure 1), counted modulo translation parallel to the confining boundaries.

The generating function of this model is

$$
\Pi(t, y)=\sum_{n=0}^{\infty} \sum_{w=0}^{n / 2} \pi_{n}(w) y^{w} t^{n},
$$

where $\pi_{n}(w)=\sum_{v} p_{n}(v, w)$. If $0 \leq y \leq 1$, by monotonicity,

$$
G(t)=\Pi(t, 1) \geq \Pi(t, y),
$$

where $G(t)$ is the generating function of polygons in a half-lattice. Then it is known that $G(t)$ is singular at $t=t_{c}=\frac{1}{\mu}=e^{-\kappa_{d}}$.

Suppose that $d \geq 3$. Then it is known

$$
\lim _{n \rightarrow \infty} n^{-1} \log \pi_{n}(w)=\log \mu_{w}
$$

exists where $\mu_{w}$ is the growth constant for self-avoiding walks in a slab of width $w$ [18]. It is proved there that $\mu_{w}<\mu_{w+1}$ and $\lim _{w \rightarrow \infty} \mu_{w}=\mu$. $\Pi(t, y)$ is bounded from below by any term in its defining series:

$$
\Pi(t, y) \geq y^{w} \sum_{n=2 w}^{\infty} \pi_{n}(w) t^{n}=y^{w} G_{w}(t) .
$$

$G_{w}(t)$ is singular at $t_{w}=\frac{1}{\mu_{w}}$.

The above gives

$$
G(t) \geq \Pi(t, y) \geq y^{w} G_{w}(t), \quad \text { if } y \leq 1, \text { for every } w>0 .
$$

The generating functions $G(t)$ and $G_{w}(t)$ have radius of convergence $\frac{1}{\mu}$ and $\frac{1}{\mu_{w}}$ respectively, where $\mu_{w}<\mu$. This shows, that for any fixed $0<y \leq 1$, the radius of convergence of $\Pi(t, y)$ is $\frac{1}{\mu_{0}(y)}$, where

$$
\mu \geq \mu_{0}(y) \geq \mu_{w} .
$$

Since $\mu_{w} \nearrow \mu$ as $w \rightarrow \infty$, this shows that

$$
\mu_{0}(y)=\mu, \quad \text { for all } 0<y \leq 1
$$

for all $d \geq 3$.

It remains to consider the case of $d=2$. There we know that the growth constant $\mu_{w}^{0}$ for polygons in a slab of width $w$ is strictly less than that of walks in a slab of width $w$, i.e. $\mu_{w}^{0}<\mu_{w}$ for all $w<\infty$ [43]. The same argument shows that

$$
\mu \geq \mu_{0}(y) \geq \mu_{w}^{0}
$$

and we give some properties of $\mu_{w}^{0}$ in the next theorem.

Theorem 3 For polygons in a two dimensional slit $\mu_{w}^{0}$ is an increasing function of $w$ and $\sup _{w} \mu_{w}^{0}=\mu$. 
Proof: Every polygon with $n$ edges and span $w$ can be converted to a polygon with $n+2$ edges and span $w+1$ so $\pi_{n+2}(w+1) \geq \pi_{n}(w)$, from which we have $\mu_{w+1}^{0} \geq \mu_{w}^{0}$. The existence of the limit $\lim _{w \rightarrow \infty} \mu_{w}^{0}$ and the fact that it is equal to $\sup _{w} \mu_{w}^{0}$ and that this in turn is equal to $\mu$ is a consequence of the arguments in Sections 4 and 6 of [18].

Since $\sup _{w} \mu_{w}^{0}=\mu$ (by the above Theorem) it follows from (4.11) that $\mu_{0}(y)=\mu$ for all $y \leq 1$ in two dimensions, and therefore for all $d \geq 2$.

\subsection{Polygons pulled at a middle vertex}

Consider $n$-edge polygons ( $n$ is automatically even) with at least one vertex in the adsorbing plane and with all vertices in or on one side of this plane. Translate the polygon so that the lexicographically first vertex in the surface is at the origin. The middle vertex is the vertex joined to the origin by two sub-walks each of length $n / 2$.

Let $c_{n}^{+}(h)$ be the number of positive walks from the origin with endpoint at height $h$ above the adsorbing plane, and $p_{n}^{+}(h)$ be the number of polygons with middle vertex at height $h$. Note that $\sum_{h} c_{n}^{+}(h)=c_{n}^{+}$. Let $C_{n}^{+}(y)$ be the partition function of $c_{n}^{+}(h)$ and $P_{n}^{+}(y)$ be the partition function of $p_{n}^{+}(h)$, with growth constants $\mu_{c}^{+}(y)$ and $\mu_{p}^{+}(y)$. The free energies are $\lambda^{+}(y)=\log \mu_{c}^{+}(y)$ and $\lambda_{p}^{+}(y)=\log \mu_{p}^{+}(y)$. The partition function $P_{n}^{+}(y)$ is the partition function of polygons pulled at their middle vertex and $C_{n}^{+}(y)$ is the corresponding partition function for walks pulled at their last vertex.

We define the generating functions

$$
W^{+}(t, y)=\sum_{n} C_{n}^{+}(y) t^{n} \quad P^{+}(t, y)=\sum_{n} P_{n}^{+}(y) t^{n}
$$

and we write their radii of convergence as $t_{c}^{+}(y)$ and $t_{p}^{+}(y)$.

We now define similar quantities for bridges. A bridge is a positive walk that takes its first step away from the adsorbing plane, never returns to the adsorbing plane and whose last vertex is in the top plane of the walk. Let $b_{n}(h)$ be the number of $n$-edge bridges of height $h$. The partition function and generating function are defined as

$$
B_{n}(y)=\sum_{h} b_{n}(h) y^{h}, \quad B(t, y)=\sum_{n} B_{n}(y) t^{n} .
$$

A bridge can be doubly unfolded in the first coordinate direction so that the origin is left-most and the first coordinate of the last vertex is right-most [17]. See Figure 3.

Let $b_{n}^{\dagger}(h)$ be the number of doubly unfolded bridges with $n$ edges and height $h$. Then

$$
b_{n}^{\dagger}(h) \leq b_{n}(h) \leq e^{o(n)} b_{n}^{\dagger}(h) .
$$

Their partition functions are related by the inequalities,

$$
B_{n}^{\dagger}(y) \leq B_{n}(y) \leq e^{o(n)} B_{n}^{\dagger}(y),
$$

and so $\lim _{n \rightarrow \infty}\left(B_{n}^{\dagger}(y)\right)^{1 / n}=\mu_{c}^{B}(y)$ where $\mu_{c}^{B}(y)=1 / t_{c}^{B}(y)=1 / t_{c}^{+}(y)$. This last equality follows from Lemma 1 and Theorem 3 in [31]. 


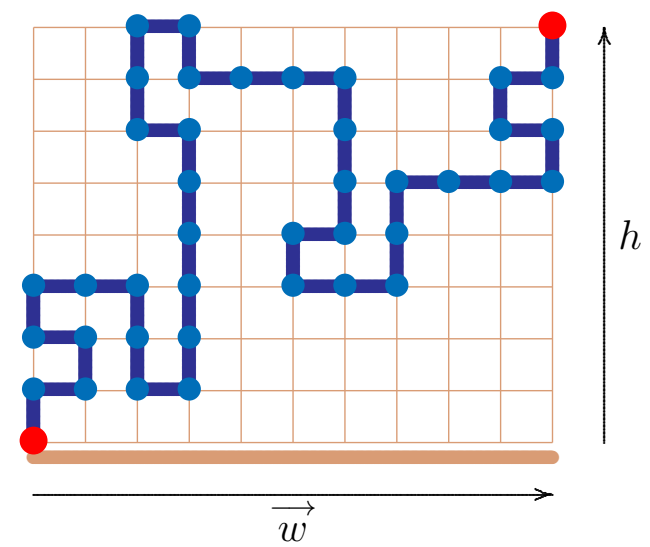

Figure 3. A doubly unfolded bridge. The vertex in the surface is in the left-most plane and the last vertex is in the right-most plane. The bridge steps away from the surface at its first step and never returns.

\subsection{A connection between polygon and walk partition functions}

Clearly $t_{c}^{+}(y) \leq t_{p}^{+}(y)$ for all $y>0$ and $t_{c}^{+}(1)=t_{p}^{+}(1)=\mu^{-1}$. If $y>1$ then $P_{n}^{+}(y) \leq p_{n} y^{n / 2}$ and $C_{n}^{+}(y) \geq y^{n}$. Taking powers $1 / n$ and then letting $n \rightarrow \infty$ gives $\mu_{p}^{+}(y) \leq \mu \sqrt{y}<y \leq \mu_{c}^{+}(y)$ for $y>\mu^{2}$, or

$$
t_{p}^{+}(y)>t_{c}^{+}(y), \quad \text { for } y>\mu^{2} .
$$

We next show how this inequality can be strengthened.

\section{Theorem 4}

$$
t_{p}^{+}\left(y^{2}\right) \geq t_{c}^{+}(y), \text { for all } y \geq 1
$$

Proof: By cutting the polygon at its middle vertex (where the force is applied) into two walks, $p_{n}^{+}(h) \leq\left(c_{n / 2}^{+}(h)\right)^{2}$, so that

$$
P_{n}^{+}(y)=\sum_{h} p_{n}^{+}(h) y^{h} \leq \sum_{h}\left(c_{n / 2}^{+}(h) y^{h / 2}\right)^{2} \leq\left(\sum_{h} c_{n / 2}^{+}(h) y^{h / 2}\right)^{2}=\left(C_{n / 2}^{+}(\sqrt{y})\right)^{2} .
$$

Take the power $1 / n$ and let $n \rightarrow \infty$ to obtain $\mu_{p}^{+}(y) \leq \mu_{c}^{+}(\sqrt{y})$. That is

$$
t_{p}^{+}\left(y^{2}\right) \geq t_{c}^{+}(y), \quad \text { for } y \geq 1 .
$$

A corollary of this Theorem is as follows: The free energy of pulled walks is $-\log t_{c}^{+}(y)$, and this is strictly increasing with $y>1$, since the model is ballistic. That is, $t_{c}^{+}(y)>t_{c}^{+}\left(y^{2}\right)$ if $y>1$. This shows with the above that $t_{p}^{+}\left(y^{2}\right) \geq t_{c}^{+}(y)>t_{c}^{+}\left(y^{2}\right)$ for $y>1$, or $t_{p}^{+}(y)>t_{c}^{+}(y)$ whenever $y>1$. This strengthens (4.15).

We next prove the corresponding inequality in the other direction. We obtain a lower bound by constructing polygons by concatenating four doubly unfolded bridges. The unfolded bridge has a width $\vec{w}$ and a height $h$, where $\vec{w}$ is the vector of widths in all except the vertical direction, and may look as illustrated in Figure 3. 


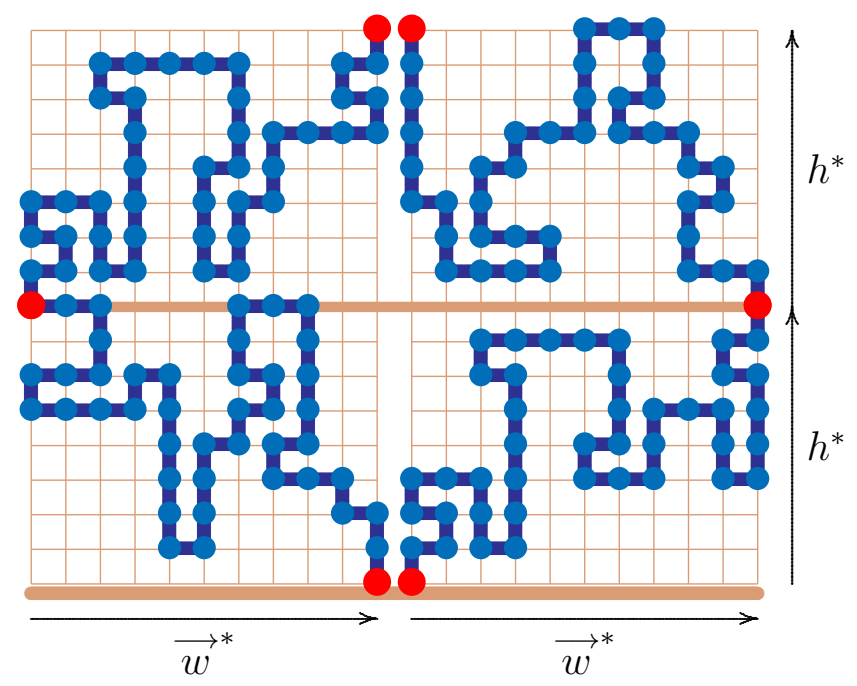

Figure 4. Four doubly unfolded bridges can be concatenated to form a polygon. The bridges are selected so that their heights and widths have their most popular values.

Next, we have to choose values for $h$ and $\vec{w}$. Let $b_{n}^{\dagger}(h, \vec{w})$ be the number of $n$-edge doubly unfolded bridges with width $\vec{w}$ and height $h$. In the partition function for doubly unfolded bridges, $B_{n}^{\dagger}(y)=\sum_{h, \vec{w}} b_{n}^{\dagger}(h, \vec{w}) y^{h}$ there are most popular values of $h$ and $\vec{w}$ (these are functions of $y$ ); denote them by $h^{*}$ and $\vec{w}^{*}$. Then $b_{n}^{\dagger}\left(h^{*}, \vec{w}^{*}\right) y^{h^{*}}$ is a largest term in $B_{n}^{\dagger}(y)=\sum_{h, \vec{w}} b_{n}^{\dagger}(h, \vec{w}) y^{h}$, so that

$$
b_{n}^{\dagger}\left(h^{*}, \vec{w}^{*}\right) y^{h^{*}} \leq B_{n}^{\dagger}(y) \leq(n+1)^{d} b_{n}^{\dagger}\left(h^{*}, \vec{w}^{*}\right) y^{h^{*}} .
$$

Taking powers $1 / n$, letting $n \rightarrow \infty$, and noting that $\left(B_{n}^{\dagger}(y)\right)^{1 / n} \rightarrow \mu_{c}^{B}(y)$, it follows that

$$
\lim _{n \rightarrow \infty}\left(b_{n}^{\dagger}\left(h^{*}, \vec{w}^{*}\right) y^{h^{*}}\right)^{1 / n}=\mu_{c}^{B}(y)=1 / t_{c}^{B}(y)=1 / t_{c}^{+}(y) .
$$

\section{Theorem 5}

$$
t_{p}^{+}\left(y^{2}\right)=t_{c}^{+}(y) \quad \text { for all } y \geq 1 .
$$

Proof: Because of Theorem 4 above we only need to prove an inequality in one direction. By reflecting and rotating unfolded bridges of most popular widths and heights they can be concatenated to form a polygon as in Figure 4.

This arrangement gives a lower bound on polygons of height $2 h^{*}$ and of length $4 n+2$ which gives a lower bound on the polygon partition function:

$$
\left(b_{n}^{\dagger}\left(h^{*}, \vec{w}^{*}\right) y^{h^{*}}\right)^{4} \leq p_{4 n+2}^{+}\left(2 h^{*}\right) y^{4 h^{*}} \leq P_{4 n+2}^{+}\left(y^{2}\right) .
$$

Take the power $1 / 4 n$ and then let $n \rightarrow \infty$. The left hand side goes to $1 / t_{c}^{+}(y)$, and the right hand side goes to $\mu_{p}^{+}\left(y^{2}\right)=1 / t_{p}^{+}\left(y^{2}\right)$. This shows that

$$
t_{p}^{+}\left(y^{2}\right) \leq t_{c}^{+}(y)
$$

Since we already know that $t_{p}^{+}\left(y^{2}\right) \geq t_{c}^{+}(y)$ the result is that

$$
t_{p}^{+}\left(y^{2}\right)=t_{c}^{+}(y) \text {. }
$$

This shows that polygons also become ballistic at $y_{c}=1$. 


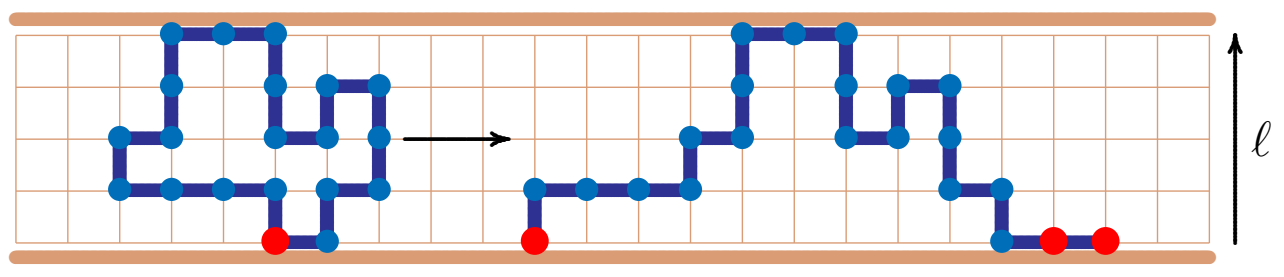

Figure 5. A polygon in a slit or slab unfolded to form a loop.

We turn our attention now to the case where $y \leq 1$. The construction in Figure 4, together with equations (4.17) and (4.19), shows that

$$
\begin{aligned}
\frac{1}{(n+1)^{4 d}}\left(B_{n}^{\dagger}(y)\right)^{4} & \leq\left(b_{n}^{\dagger}\left(h^{*}, \vec{w}^{*}\right) y^{h^{*}}\right)^{4} \\
& \leq p_{4 n+2}^{+}\left(2 h^{*}\right) y^{4 h^{*}} \leq P_{4 n+2}^{+}\left(y^{2}\right) .
\end{aligned}
$$

Recall that $h^{*}$ and $\vec{w}^{*}$ are the most popular values of the height and width. We know that, for $y \leq 1, t_{c}^{+}(y)=t_{c}^{B}(y)=1 / \mu[31]$. Hence $1 / \mu \geq t_{p}^{+}\left(y^{2}\right) \geq t_{c}^{+}\left(y^{2}\right)=1 / \mu$ for all $y \leq 1$. This proves the following theorem:

Theorem 6 When $y \leq 1, t_{p}^{+}(y)=\frac{1}{\mu}$.

\section{Polygons pulled from their top plane}

Define $p_{n}(\ell)$ to be the number of polygons, with at least one vertex in $x_{d}=0$ and with highest vertices at height $\ell$. A model of pulled polygons, where the highest vertices are pulled vertically by a force $f$, is defined by the partition function

$$
P_{n}(y)=\sum_{\ell \geq 0} p_{n}(\ell) y^{\ell}
$$

Here, the activity $y=e^{f / k_{B} T}$ is introduced and is equal to the exponential of the reduced pulling force. The generating function of this model is given by $P(t, y)$ and its radius of convergence is denoted $t_{p}(y)$.

It remains to relate the limiting free energy of this model to that of polygons pulled in the middle, considered above. Note that the midpoint of a polygon of height $\ell$ is itself at most at height $\ell$. Thus, assuming that $y \geq 1, P_{n}^{+}(y) \leq P_{n}(y)$. This shows that

$$
\lim _{n \rightarrow \infty} \frac{1}{n} \log P_{n}^{+}(y) \leq \liminf _{n \rightarrow \infty} \frac{1}{n} \log P_{n}(y) .
$$

This, in particular, shows that $t_{c}^{+}(\sqrt{y})=t_{p}^{+}(y) \geq t_{p}(y)$.

Existence of the free energy will now be shown by bounding the limiting supremum.

Cut the polygon at its lexicographically first vertex in the surface, and unfold it into a bridge in the $x_{1}$-direction (by adding a single edge in the horizontal direction). This is schematically illustrated in Figure 5.

The polygon is unfolded into a loop of height $\ell$. Denote the partition function of these (unfolded) loops by $L_{n}^{\ddagger}(y)$, and it follows that $P_{n}(y) \leq e^{o(n)} L_{n}^{\ddagger}(y)$. Let the number of unfolded loops of length $n$ and height $\ell$ be denoted by $l_{n}^{\ddagger}(\ell)$. Then

$$
L_{n}^{\ddagger}(y)=\sum_{\ell=0}^{n / 2} l_{n}^{\ddagger}(\ell) y^{\ell} .
$$



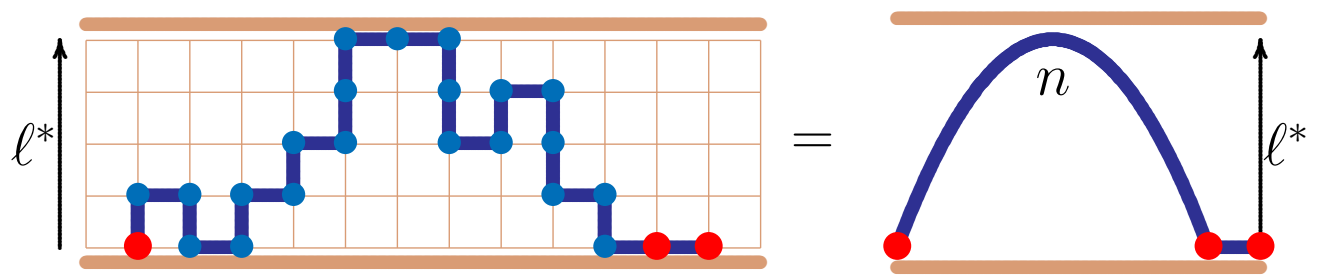

Figure 6. An unfolded loop with its last step in the surface and with height equal to the most popular height $\ell^{*}$. On the right is a schematic diagram of this class of loops.

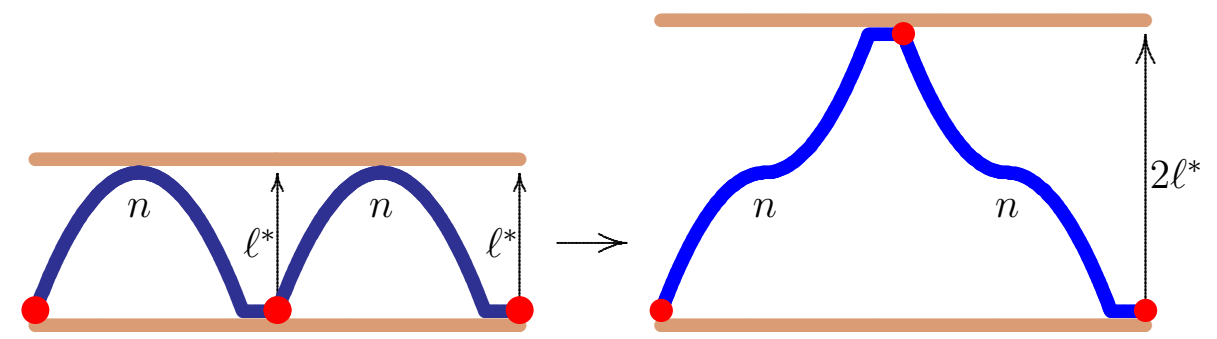

Figure 7. Two unfolded loops are concatenated in a slab of height $\ell^{*}$ and then reflected through the top boundary of the slab to obtain a loop in a slab of height $2 \ell^{*}$.

For each value of $y>0$ there is a most popular value of $\ell$ in this summation, and this is denoted by $\ell^{*}$ (this is dependent on $y$ and on $n$ ). In particular,

$$
l_{n}^{\ddagger}\left(\ell^{*}\right) y^{\ell^{*}} \leq L_{n}^{\ddagger}(y) \leq \frac{1}{2} n l_{n}^{\ddagger}\left(\ell^{*}\right) y^{\ell^{*}} .
$$

The loops in this most popular class are schematically illustrated in Figure 6.

Two loops in this most popular class can be concatenated as illustrated schematically in Figure 7. If the middle part of the concatenated loops is reflected through the top plane as shown, then a loop of height $2 \ell^{*}$ is obtained with the property that its middle vertex is also in the top plane. This loop consists of two self-avoiding walks of length $n$ and height $2 \ell^{*}$. Thus

$$
\begin{aligned}
\frac{4}{n^{2}} L_{n}^{\ddagger 2}(y) & \leq\left(l_{n}^{\ddagger}\left(\ell^{*}\right) y^{\ell^{*}}\right)^{2} \leq\left(c_{n}^{+}\left(2 \ell^{*}\right) y^{\ell^{*}}\right)^{2} \\
& \leq \sum_{\ell}\left(c_{n}^{+}(\ell) y^{\ell / 2}\right)^{2} \leq\left(\sum_{\ell} c_{n}^{+}(\ell) y^{\ell / 2}\right)^{2}=\left(C_{n}^{+}(\sqrt{y})\right)^{2} .
\end{aligned}
$$

That is, since $P_{n}(y) \leq e^{o(n)} L_{n}^{\ddagger}(y)$,

$$
\limsup _{n \rightarrow \infty} \frac{1}{n} \log P_{n}(y) \leq \lim _{n \rightarrow \infty} \frac{1}{n} \log C_{n}^{+}(\sqrt{y})
$$

with the result that by equation (5.2),

$$
\begin{aligned}
-\log t_{p}^{+}(y) & =\lim _{n \rightarrow \infty} \frac{1}{n} \log P_{n}^{+}(y) \leq \liminf _{n \rightarrow \infty} \frac{1}{n} \log P_{n}(y) \\
& \leq \limsup _{n \rightarrow \infty} \frac{1}{n} \log P_{n}(y) \leq \lim _{n \rightarrow \infty} \frac{1}{n} \log C_{n}^{+}(\sqrt{y}) \\
& =-\log t_{c}^{+}(\sqrt{y})=-\log t_{p}^{+}(y) .
\end{aligned}
$$

Here, recall that $P_{n}^{+}(y)$ is the partition function of polygons pulled in the middle, $P_{n}(y)$ is the partition function of polygons pulled in their highest plane, and $C_{n}^{+}(\sqrt{y})$ is the partition function of walks pulled at their endpoint, and $y \geq 1$. 
By equation (4.20) this shows that for polygons pulled in their top plane,

$$
\lim _{n \rightarrow \infty} \frac{1}{n} \log P_{n}(y)=-\log t_{c}^{+}(\sqrt{y})=-\log t_{p}^{+}(y) .
$$

That is, the free energy of polygons pulled at their middle point is equal to the free energy of polygons pulled in their top plane if $y \geq 1$. The case of $y \leq 1$ follows from Figure 4 and the proof of Theorem 6. This gives the following theorem:

Theorem 7

$$
\lambda_{0}(y)=\lim _{n \rightarrow \infty} \frac{1}{n} \log P_{n}(y)=-\log t_{c}^{+}(\sqrt{y})=-\log t_{p}^{+}(y)
$$

for all $y>0$. When $y \leq 1$ this is equal to $\log \mu$.

For polygons, as well as for walks, there is a phase transition to a ballistic phase at $y=1$ but the response of polygons and walks is different in this ballistic phase.

\section{Polygons pulled from an interacting surface}

In this section we consider the full problem where the polygon interacts with the surface $(a \neq 1)$ and the applied force is pulling the polymer off the surface in its top plane $(y>1)$. We derive some results about the $a$-dependence of the free energy at fixed $y>1$ and show that there is a phase transition from an adsorbed phase to a ballistic phase at $a=a_{c}^{0}(y)$.

First consider the situation when $y>0$ and $a \leq 1$.

Theorem 8 For $y>0$ and $a \leq 1$ the thermodynamic limit

$$
\lim _{n \rightarrow \infty} n^{-1} \log P_{n}(a, y) \equiv \psi_{0}(a, y)
$$

exists so we have a well defined limiting free energy. Moreover, in this region of the (a,y)-plane the free energy is independent of a so that $\psi_{0}(a, y)=\psi_{0}(1, y)=\lambda_{0}(y)$.

Proof: For fixed $y>0$ and for all $a \leq 1$, by monotonicity

$$
P_{n}(0, y) \leq P_{n}(a, y) \leq P_{n}(1, y) .
$$

Consider an $n$-edge polygon $\omega$ with span $s$. Suppose that $V(\omega)$ is the vertex of $\omega$ in the surface (i.e. that is a visit) with lexicographically first coordinates in the surface. When $d=2$ there is exactly one edge of $\omega$ in the surface that is incident on $V$. For $d>2$, if there are two such edges, choose the one with lexicographically first mid-point. Delete this edge in the surface, incident on $V$, translate $\omega$ by unit distance away from the surface, add two edges to connect the resulting walk to the surface and add an edge in the surface to obtain a polygon $\omega^{\prime}$ with $n+2$ edges and span $s+1$ with exactly two visits.

$$
\sum_{v} p_{n}(v, s) \leq p_{n+2}(0, s+1) .
$$

This implies that

$$
P_{n}(1, y) \leq \frac{1}{y} P_{n+2}(0, y)
$$

and hence, from equation (6.2),

$$
y P_{n-2}(1, y) \leq P_{n}(0, y) \leq P_{n}(a, y) \leq P_{n}(1, y) .
$$

Take logarithms, divide by $n$, let $n \rightarrow \infty$, and the result follows. 
When $y \geq 1$ and $a \geq 1$ we have two useful lower bounds on the partition function that follow from monotonicity, namely

$$
P_{n}(a, y) \geq P_{n}(a, 1) \quad \text { and } \quad P_{n}(a, y) \geq P_{n}(1, y) .
$$

These bounds imply that

$$
\liminf _{n \rightarrow \infty} n^{-1} \log P_{n}(a, y) \geq \max \left[\kappa_{0}(a), \lambda_{0}(y)\right] .
$$

We can also construct an upper bound similar to the one derived for the self-avoiding walk model [30]. Let $l_{n}(h)$ be the number of loops (or arches) with $n$ edges and span in the $x_{d}$-direction equal to $h$. Write the partition function as

$$
L_{n}(y)=\sum_{h} l_{n}(h) y^{h} .
$$

Theorem 9 For all $d \geq 2$

$$
P_{n}(a, y) \leq y^{-1} \sum_{m} C_{m}(a, 1) L_{n-m+2}(y)
$$

Proof: Every $n$-edge polygon with span $h$ must have at least one edge with $x_{d}=h$. Either the polygon has every edge in $x_{d}=0$ so that $h=0$, or $h \geq 1$. If $h=0$ choose the edge that is lexicographically first and add two edges to the polygon just before and just after the distinguished edge in $x_{d}=0$ to produce a unique edge in $x_{d}=1$ in a single loop of 3 edges. If $h \geq 1$ choose the edge in $x_{d}=h$ that is lexicographically first. This edge must be in a loop with, say, $n-m$ edges. The remainder of the polygon is a walk with $m$ edges. Add two edges to the loop just before and just after the distinguished edge in $x_{d}=h$ to produce a unique edge in $x_{d}=h+1$. This unique edge distinguishes the loop of length $n-m+2$ from the rest of the polygon. By noting that the rest of the polygon is a walk of length $m$, the following inequality is obtained:

$$
p_{n}(v, h) \leq \sum_{m} c_{m}(v) l_{n-m+2}(h+1)
$$

where $c_{m}(v)=\sum_{h} c_{m}^{+}(v, h)$. Now multiply both sides by $y^{h} a^{v}$ and sum over $h$ and $v$ giving (6.9) which proves the theorem.

Define the generating functions

$$
\widehat{L}(t, y)=\sum_{n} L_{n}(y) t^{n} \quad \text { and } \quad \widehat{C}(t, a)=\sum_{n} C_{n}^{+}(a, 1) t^{n} .
$$

By the convolution theorem

$$
\sum_{n} \sum_{m} C_{m}(a, 1) L_{n-m+2}(y) t^{n} \leq t^{-2} \widehat{C}(a, t) \widehat{L}(y, t)=t^{-2} \widehat{B}(a, y, t) .
$$

The radius of convergence of $\widehat{C}(t, a)$ is $t_{1}(a)=\exp [-\kappa(a)]$ and the radius of convergence of $\widehat{L}(t, y)$ is $t_{2}(y)$ so the radius of convergence of $\widehat{B}(a, y, z)$ is $\min \left[t_{1}(a), t_{2}(y)\right]$. This implies that

$$
\limsup _{n \rightarrow \infty} n^{-1} \log P_{n}(a, y) \leq \max \left[\kappa(a), \liminf _{n \rightarrow \infty} n^{-1} \log L_{n}(y)\right]
$$

or, roughly, the free energy of pulled polygons interacting with the surface is bounded above by the maximum of the free energy of walks interacting with the surface and pulled loops.

By the results in Section 5 (see equation (5.5)) it follows

$$
e^{o(n)} P_{n}(y) \leq L_{n}^{\ddagger}(y) \leq e^{o(n)} C_{n}^{+}(\sqrt{y})=e^{o(n)} P_{n}(y) .
$$


Taking logarithms, dividing by $n$, and letting $n \rightarrow \infty$, it follows that

$$
\lim _{n \rightarrow \infty} \frac{1}{n} \log L_{n}^{\ddagger}(y)=-\log t_{p}(y) .
$$

But, by unfolding loops, it follows that $e^{o(n)} L_{n}(y) \leq L_{n}^{\ddagger}(y) \leq L_{n}(y)$. Thus

$$
\lim _{n \rightarrow \infty} \frac{1}{n} \log L_{n}(y)=-\log t_{p}(y) .
$$

In equation (6.13) this gives

$$
\limsup _{n \rightarrow \infty} n^{-1} \log P_{n}(a, y) \leq \max \left[\kappa(a), \lambda_{0}(y)\right]
$$

When $d>2$ we know that $\kappa_{0}(a)=\kappa(a)$ [42] so the above result can be replaced with

$$
\lim _{n \rightarrow \infty} n^{-1} \log P_{n}(a, y)=\max \left[\kappa_{0}(a), \lambda_{0}(y)\right], \quad d \geq 3 .
$$

This gives a complete characterization of the phase boundary when $d \geq 3$ and we state this as a theorem.

Theorem 10 When $d \geq 3$ the phase boundary between the ballistic and adsorbed phases for $y \geq 1$ is determined by the solution of the equation $\kappa_{0}(a)=\lambda_{0}(y)$.

Proof: This follows immediately from (6.17).

Since $\kappa_{0}(a)=\kappa(a)[42]$ and $\lambda_{0}(y)=\lambda(\sqrt{y})$ the phase boundary is determined by the properties of the self-avoiding walk problem. We know the asymptotics of both $\kappa(a)[40]$ and $\lambda(y)$ [30] so we know the behaviour of the phase boundary for polygons at large values of $a$. We can switch into the force-temperature plane and this corresponds to the behaviour at small values of the temperature. In particular, the critical force - temperature curve is reentrant for all $d>2$. The phase transition between the ballistic and adsorbed phases is first order, except perhaps at $\left(a_{c}^{0}, 1\right)$. This follows mutatis mutandis from the arguments in [13] for the self-avoiding walk model.

When $d=2$ we know that

$$
\begin{aligned}
\max \left[\kappa_{0}(a), \lambda_{0}(y)\right] & \leq \liminf _{n \rightarrow \infty} n^{-1} \log P_{n}(a, y) \\
& \leq \limsup _{n \rightarrow \infty} n^{-1} \log P_{n}(a, y) \leq \max \left[\kappa(a), \lambda_{0}(y)\right] .
\end{aligned}
$$

Unlike the self-avoiding walk problem [30] we do not have a precise condition for locating the phase boundary when $d=2$, since we only have lower and upper bounds on the free energy when $a>1$ and $y>1$.

\section{Pushing a polygon towards an interacting surface}

We now consider the situation when $d \geq 3$ and when $0 \leq a \leq a_{c}^{0}$ and $0<y \leq 1$. First consider $0 \leq a \leq 1$ and $0<y \leq 1$. By theorem 8 we know that

$$
\psi_{0}(a, y)=\psi_{0}(1, y) .
$$

By theorem 7 it follows that $\lambda_{0}(y)=\psi_{0}(1, y)=\psi(1, \sqrt{y})=\lambda(\sqrt{y})$ (see the discussion of the walk problem in the introduction). But $\lambda(\sqrt{y})=\log \mu$ for $0<y \leq 1$ [31]. Thus $\psi_{0}(a, y)=\log \mu$ for $0 \leq a \leq 1$ and $0<y \leq 1$

Since $\psi_{0}(a, y)$ is convex in each of its variables, the critical curve $y_{c}(a)$ in Figure 2 is a nondecreasing function of $a$. We now show why $y_{c}(a)$ has a jump discontinuity at $a=a_{c}^{0}$ so that the phase boundary between the free and adsorbed phases is a vertical line segment in Figure 2. Take 
$a=a_{c}^{0}-\epsilon$ for any $\epsilon>0$. At $y=1, \psi_{0}(a, 1)=\log \mu$. But $\psi_{0}(a, y)$ is monotone non-decreasing in $y$ so it cannot be greater than $\log \mu$ for $y<1$, so that $\psi_{0}(a, y)=\log \mu$ since it cannot be smaller than $\log \mu$.

We now give an alternative proof that the phase boundary between the free and adsorbed phases is a vertical line segment in Figure 2. In fact this argument proves considerably more and essentially completes our knowledge of the phase diagram when $d \geq 3$. We shall need some preliminary lemmas. Let $\pi_{n}^{w}(a)=\sum_{v} p_{n}(v, w) a^{v}$. By a concatenation argument we can show that the limit

$$
\kappa_{0}^{w}(a)=\lim _{n \rightarrow \infty} n^{-1} \log \pi_{n}^{w}(a)
$$

exists. For instance, one can use a modification of the concatenation construction used in Section 4 of [18] coupled with a generalized supermultiplicative inequality. Let $\widehat{\pi}_{n}^{w}(a)=\sum_{u \leq w} \pi_{n}^{u}(a)$. This sum includes all polygons with span at most $w$.

Lemma 1 When $d \geq 2, \kappa_{0}^{w}(a) \leq \kappa_{0}^{w+1}(a)$.

Proof: Each polygon contributing to the sum $\pi_{n}^{w}(a)=\sum_{v} p_{n}(v, w) a^{v}$ has at least one edge in the hyperplane $x_{d}=w$. If there is more than one such edge, take the one with lexicographically first midpoint. Translate this edge unit distance into the hyperplane $x_{d}=w+1$ and add two edges to reconnect the polygon. Then

$$
\pi_{n}^{w}(a) \leq \pi_{n+2}^{w+1}(a)
$$

and taking logarithms, dividing by $n$ and letting $n \rightarrow \infty$ completes the proof.

Lemma 2 The exponential growth rate of $\widehat{\pi}_{n}^{w}(a)$ is identical to that of $\pi_{n}^{w}(a)$ for all $w<\infty$.

Proof: The result follows from the following inequalities:

$$
\pi_{n}^{w}(a) \leq \widehat{\pi}_{n}^{w}(a) \leq(w+1) \pi_{n}^{w}(a)=\exp \left[\kappa_{0}^{w}(a) n+o(n)\right] .
$$

Lemma 3 When $d \geq 2, \sup _{w} \kappa_{0}^{w}(a)=\kappa_{0}(a)$.

Proof: Clearly $\kappa_{0}^{w}(a) \leq \kappa_{0}(a)$ for all $w$. Write $n=N r+q, 0 \leq q<N$. Concatenate $r$ polygons each with $N$ edges, and a final polygon with $q$ edges, using the concatenation construction detailed in Section 4 of [18]. Each polygon with $N$ edges has span no larger than $w=N / 2$ so the resulting polygon (with $N r+q$ edges) has span no larger than $w=N / 2$. By the argument leading to (4.14) in [18] this gives

$$
n^{-1} \log \widehat{\pi}_{n}^{w}(a) \geq N^{-1}(1-q / n) \log P_{N}(a, 1)-2 N^{-1}(1-q / N) \log \left[(d-1) N^{d-1}\right] .
$$

Hence

$$
\kappa_{0}^{w}(a) \geq N^{-1} \log P_{N}(a, 1),
$$

where we recall that $w=N / 2$. As $N \rightarrow \infty$ the right hand side goes to $\kappa_{0}(a) \operatorname{sosup}_{w} \kappa_{0}^{w}(a) \geq \kappa_{0}(a)$ which completes the proof.

This allows us to prove the following:

Theorem 11 When $d \geq 2$

$$
\psi_{0}(a, y)=\psi_{0}(a, 1)=\kappa_{0}(a)
$$

for all $0<y \leq 1$. 
Proof: Fix $0<y \leq 1$. By monotonicity $P_{n}(a, y) \leq P_{n}(a, 1)$ so

$$
\limsup _{n \rightarrow \infty} n^{-1} \log P_{n}(a, y) \leq \kappa_{0}(a) .
$$

By considering one term in the partition function

$$
P_{n}(a, y) \geq y^{w} \sum_{v} p_{n}(v, w) a^{v}=\pi_{n}^{w}(a)
$$

and

$$
\liminf _{n \rightarrow \infty} n^{-1} \log P_{n}(a, y) \geq \lim _{n \rightarrow \infty} n^{-1} \log \pi_{n}^{w}(a)=\kappa_{0}^{w}(a)
$$

for all $w>0$. Hence

$$
\liminf _{n \rightarrow \infty} n^{-1} \log P_{n}(a, y) \geq \sup _{w} \kappa_{0}^{w}(a)=\kappa_{0}(a)
$$

Then (7.7) and (7.10) prove the theorem.

In particular, this proves that the phase boundary between the free and adsorbed phases is a vertical line in the $(a, y)$-phase diagram, at $a=a_{c}^{0}$.

\section{The phase diagram}

In this section we give a brief summary of what is known rigorously about the form of the phase diagram in the $(a, y)$-plane.

There is a free phase when $a<a_{c}^{0}$ and $y<1$. When $a>a_{c}^{0}$ and $y<1$ the system is in an adsorbed phase and $\psi^{0}(a, y)=\kappa^{0}(a)$, independent of $y$.

Suppose that $y_{I}(a)$ is the solution of the equation $\lambda_{0}(y)=\kappa(a)$ and suppose that $y_{I I}(a)$ is the solution of the equation $\lambda_{0}(y)=\kappa_{0}(a)$. When $d \geq 3 \kappa_{0}(a)=\kappa(a)$ so $y_{I}(a)=y_{I I}(a)$. For this case $(d \geq 3)$ there is a ballistic phase (where $\left.\psi_{0}(a, y)=\lambda_{0}(y)\right)$ when $y>\max \left[1, y_{I}(a)\right]$ and an adsorbed phase (where $\left.\psi_{0}(a, y)=\kappa_{0}(a)\right)$ when $a>a_{c}^{0}$ and $y<y_{I}(a)$.

When $d=2$ we know less. The system is in a ballistic phase when $y>\max \left[1, y_{I}(a)\right]$ but we do not know whether $y=y_{I}(a)$ is a boundary of this phase. When $y<y_{I I}(a)$ the system is no longer ballistic. There are two possibilities:

(i) The phase boundary of the ballistic phase, $y_{B}(a)$, is equal to $y_{I I}(a)$. In this case there are three phases: a free phase, a ballistic phase and an adsorbed phase (when $a>a_{c}^{0}$ and $y<y_{I I}(a)$ ).

(ii) The phase boundary of the ballistic phase satisfies $y_{I I}(a)<y_{B}(a) \leq y_{I}(a)$. Then there at least four phases: a free phase, a ballistic phase, an adsorbed phase (including the region defined by $a>a_{c}^{0}$ and $y<1$ ) and at least one additional phase where the free energy depends on both $a$ and $y$, in the region defined by $a>a_{c}^{0}$ and $1<y<y_{B}(a)$. We do not know if $y=1$ is a phase boundary.

\section{Numerical results from Monte Carlo data}

In order to investigate the phase behaviour in the half square lattice, we collected approximate enumeration data for polygons as a function of $a$ and $y$ using the GARM algorithm [39]. This algorithm was implemented using BFACF-style elementary moves [5] (see figure 8) on polygons in the half-lattice. 


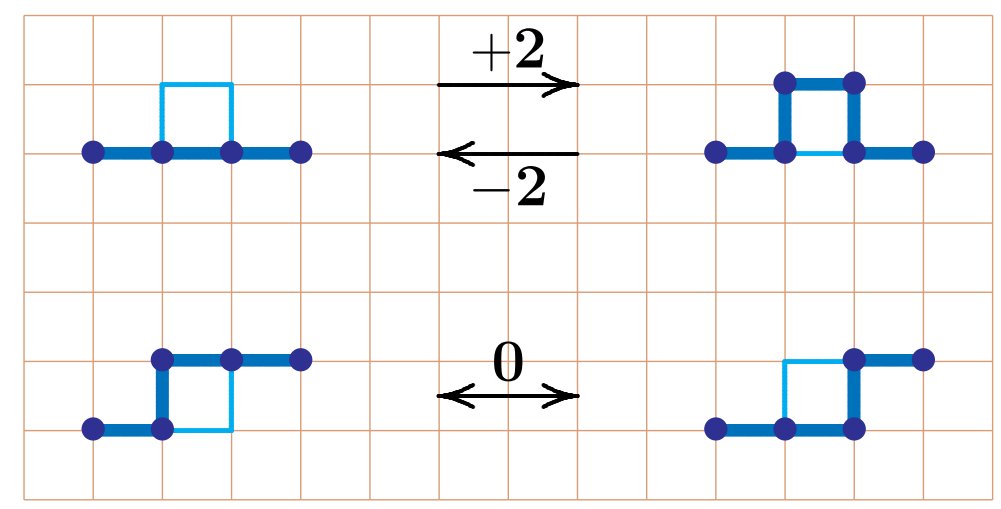

Figure 8. BFACF moves in the square lattice [5]. A positive move (or a positive BFACF move) increases the length of a polygon by 2 steps by replacing an edge with three edges. The reverse of this move reduces the length of the polygon by 2 steps and is a negative BFACF move. Neutral $B F A C F$ moves change the polygon locally as shown without changing its length.

Polygons in the upper half square lattice and constrained to have at least one edge in the boundary of the half-lattice (the $x$-axis) can be sampled by executing BFACF moves. This is done as follows using a GARM implementation of BFACF moves.

A positive BFACF move can be done along the polygon by replacing one edge by three $\longrightarrow \rightarrow$ while maintaining the constraints that the polygon has to step at least once in the $x$ axis and must stay in the upper half square lattice. The collection of all possible positive BFACF moves is the positive atmosphere of the polygon. The number of possible BFACF moves in the positive atmosphere of a polygon $\omega$ is denoted $a_{+}(\omega)$.

A negative BFACF move is the reversal of a positive BFACF move, and is implemented by replacing three edges by one: $\Pi \rightarrow \longrightarrow$, while maintaining the constraints that the polygon has to contain an edge in the $x$-axis and stay in the upper half square lattice. The collection of all possible negative BFACF moves is the negative atmosphere of the polygon. The number of possible BFACF moves in the negative atmosphere of a polygon $\omega$ is denoted $a_{-}(\omega)$.

Neutral BFACF moves are implemented by local changes involving two edges of the polygon: $\Gamma \longleftrightarrow \downarrow$ while maintaining the constraints that the polygon has to contain an edge in the $x$ axis and stay in the upper half square lattice. The collection of all possible neutral BFACF moves is the neutral atmosphere of the polygon. The number of possible BFACF moves in the neutral atmosphere of a polygon $\omega$ is denoted $a_{0}(\omega)$.

A sequence $\phi=\left\langle\phi_{0}, \phi_{1}, \phi_{2}, \ldots, \phi_{n}\right\rangle$ of polygons in the half square lattice can be sampled by executing a move uniformly selected from the positive and neutral atmospheres of $\phi_{j}$ to find $\phi_{j+1}$. The sequence is started in the polygon $\phi_{0}$ of length 4 with one edge in the $x$-axis. Notice that any polygon $\phi_{j}$ has at least one atmospheric move which can be executed on it, and that every polygon $\omega$ can be obtained in the upper half square lattice from $\phi_{0}$ by executing positive and neutral BFACF moves. (To see this, reverse the steps by starting at $\omega=\phi_{n}$, and show that it can be made shorter by executing a negative atmospheric move, sometimes after a neutral move was done a number of times).

Let $\phi_{n}$ be a state with $v$ visits and top plane of height $h$. The probability of generating a 
sequence $\phi$, starting at $\phi_{0}$ and ending in $\phi_{n}$, is given by

$$
P(\phi)=\prod_{j=0}^{n-1} \frac{1}{a_{0}\left(\phi_{j}\right)+a_{+}\left(\phi_{j}\right)} .
$$

Assign a weight

$$
W(\phi)=\prod_{j=0}^{n-1} \frac{a_{0}\left(\phi_{j}\right)+a_{+}\left(\phi_{j}\right)}{a_{0}\left(\phi_{j+1}\right)+a_{-}\left(\phi_{j+1}\right)}
$$

to the squence $\phi$.

The average weight of sequences ending in the state $\phi_{n}$ is given by

$$
\left\langle W\left(\phi_{n}\right)\right\rangle=\sum_{\phi \rightarrow \phi_{n}} P(\phi) W(\phi)=\sum_{\phi \rightarrow \phi_{n}} \prod_{j=1}^{n} \frac{1}{a_{0}\left(\phi_{j}\right)+a_{-}\left(\phi_{j}\right)} .
$$

This, however, is the probability of the reverse sequence starting in the state $\phi_{n}$ and ending in the state $\phi_{0}$ if only negative and neutral moves are done. This probability is equal to 1 , since these sequences end up in state $\phi_{0}$ with probability 1 . In other words,

$$
\left\langle W\left(\phi_{n}\right)\right\rangle=\sum_{\phi \rightarrow \phi_{n}} \prod_{j=1}^{n} \frac{1}{a_{0}\left(\phi_{j}\right)+a_{-}\left(\phi_{j}\right)}=1 .
$$

This is the GARM counting theorem [39].

If $S_{n}(v, w)$ is the set of all polygons of length $n$ in the half-lattice with $v$ visits and height $w$, then the average weight of sequences ending up in states in $S_{n}(v, w)$ is given by

$$
\begin{aligned}
W_{v, w} & =\sum_{\phi_{n} \in S(v, w)}\left\langle W\left(\phi_{n}\right)\right\rangle \\
& =\sum_{\phi_{n} \in S(v, w)} \sum_{\phi \rightarrow \phi_{n}} \prod_{j=1}^{n} \frac{1}{a_{0}\left(\phi_{j}\right)+a_{-}\left(\phi_{j}\right)}=\sum_{\phi_{n} \in S(v, h)} 1=p_{n}(v, w) .
\end{aligned}
$$

In other words, by computing the average weight $W_{v, w}$ of polygons of length $n$ with $v$ visits and height $w$, estimates of the microcanonical partition function $p_{n}(v, w)$ are obtained. This is an example of approximate enumeration [25] and these data can be used to determine average number of visits or height for polygons of fixed length.

The algorithm is implemented with pruning and enrichment in exactly the same way the PERM or flatPERM algorithms are implemented. For details, see references [10] and [38]. The resulting implementation of flatGARM is a flat histogram sampling method which continually prunes states of low weight that do not contribute much to the partition function, and otherwise enriches states of high weight in the sampling. The algorithm was run to complete about 11,000 GARM sequences and we collected data for polygons with up to 200 edges and computed the free energy, the mean number of visits and the mean height of the polygons, as well as the variances of these quantities.

In Figure 9 we show the average value of the density of visits $V_{n}$ and the average value of the scaled height $H_{n}$ plotted against $\log y$ for $a=3, a=4$ and $a=5$. There is clear evidence for two phase transitions in these figures. We know that for $\log y<0$ the density of visits and scaled height are independent of $y$. In all four panels the curves seem to be approaching horizontal lines as $n$ increases in this regime. There is a transition to a phase with a reduced number of visits and a larger value of the height around $y=1$, and a second, more marked, transition at a location whose 

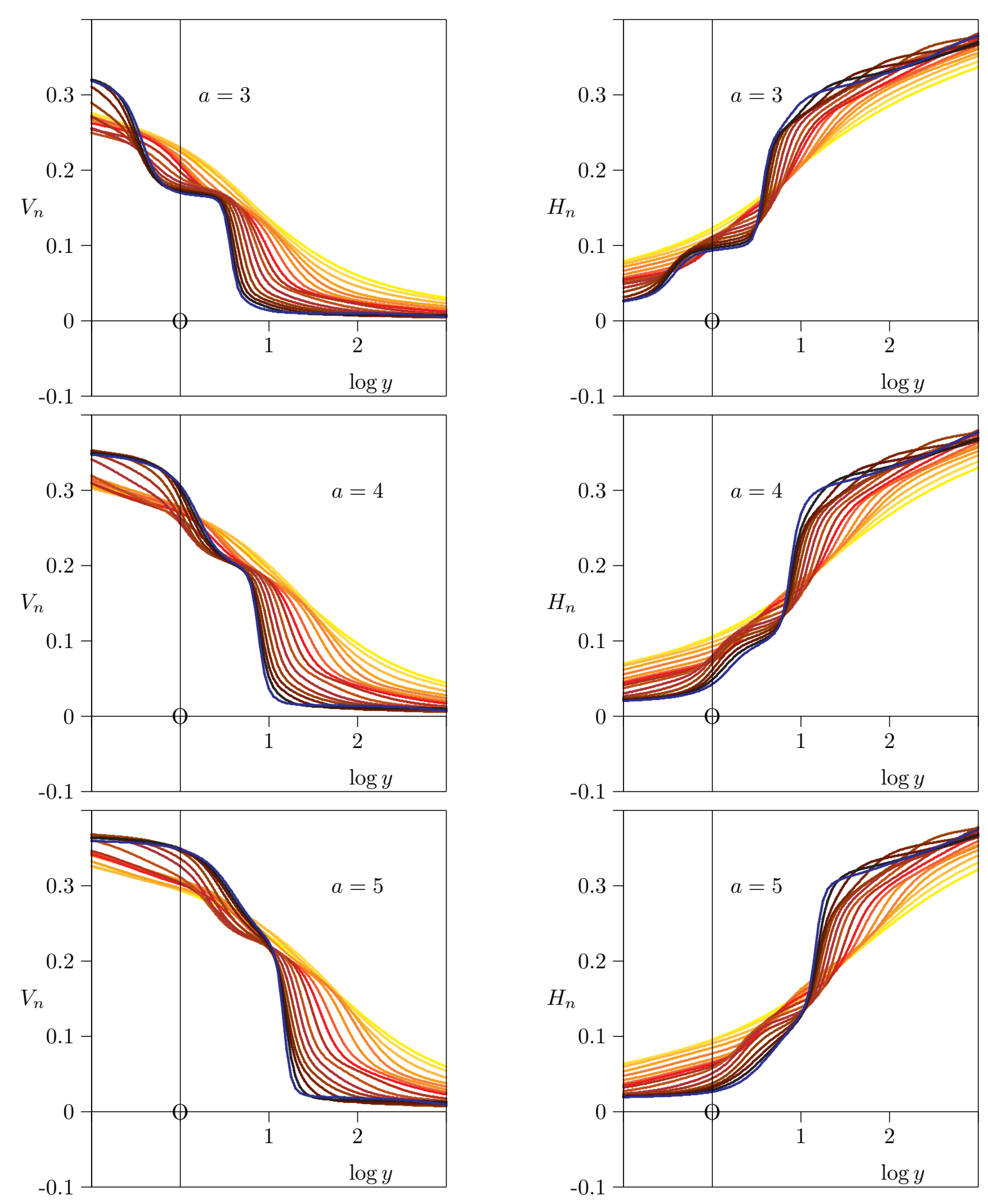

Figure 9. Energies of pulled adsorbing polygons. Left panels: The average density of visits $V_{n}$ as a function of $\log y$ for $a=3$ (top left panel), $a=4$ (middle left panel) and $a=5$ (bottom left panel). Right panels: The average height $H_{n}$ as a function of $\log y$ for $a=3$ (top right panel), $a=4$ (middle right panel) and $a=5$ (bottom right panel). In all these graphs the value of $n$ increased from 40 to 200 in steps of 10, with curves progressively darker as $n$ increases. 

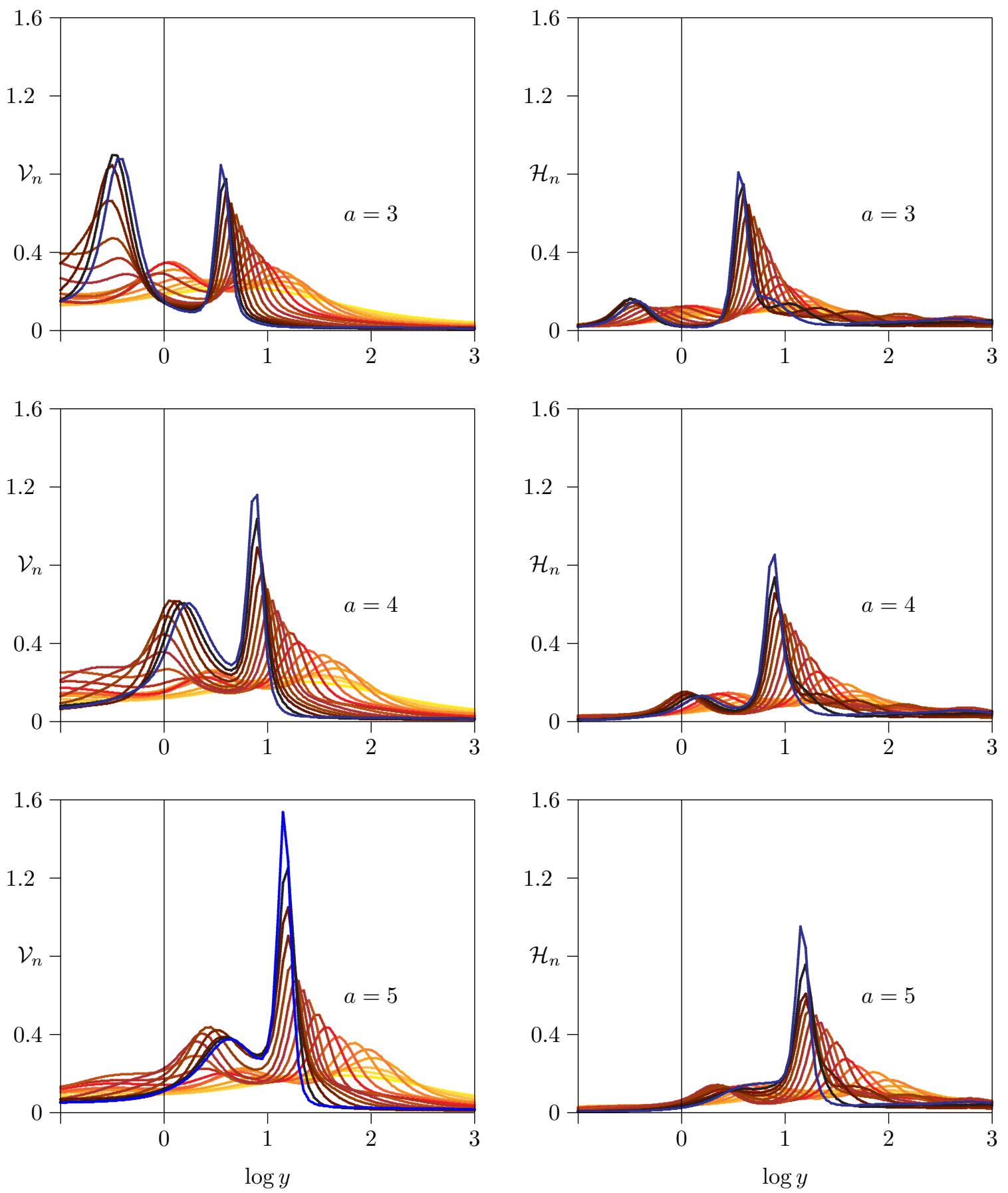

Figure 10. Variances of pulled adsorbing polygons. Left panels: The variance $\mathcal{V}_{n}$ of the density of visits as a function of $\log y$ for $a=3$ (top left panel), $a=4$ (middle left panel) and $a=5$ (bottom left panel). Right panels: The variance $\mathcal{H}_{n}$ of the scaled height as a function of $\log y$ for $a=3$ (top right panel), $a=4$ (middle right panel) and $a=5$ (bottom right panel). In all these graphs the value of $n$ increased from 40 to 200 in steps of 10, with curves progressively darker as $n$ increases. 
value depends on $a$ from this phase to a ballistic phase where the density of visits approaches zero. In the intermediate regime between the two transitions the density of visits and the average scaled height depend on both $a$ and $y$. This regime is referred to as a mixed phase.

For instance, when $a=5$, the curves for $V_{n}$ are approaching a horizontal line when $\log y \leq 0$, as we know must happen from our rigorous arguments. For $\log y>0$ there is a regime in which $V_{n}$ is a decreasing function of $\log y$ and $H_{n}$ is an increasing function of $\log y$, so the free energy depends on $y$. It also depends on $a$, as can be seen by comparing with the results at $a=3$ and $a=4$. When $\log y$ is somewhat larger than 1 there is a rapid decrease in $V_{n}$ and a rapid increase in $H_{n}$ and then at larger values of $y$ both quantities become less dependent on $y$. All of this suggests two transitions, one from an adsorbed phase where the free energy only depends on $a$ to a mixed phase where the free energy depends on both $a$ and $y$, and a second transition at larger $y$ to a ballistic phase. In this third (ballistic) phase the free energy depends on $y$ but is essentially independent of $a$. We know rigorously that in the infinite $n$ limit the free energy is independent of $a$ in the ballistic phase.

In Figure 10 we show the corresponding fluctuation quantities $\mathcal{V}_{n}$ and $\mathcal{H}_{n}$ of the data in Figure 9. In all four panels there are two peaks consistent with two phase transitions. If we look first at the transitions at larger values of $y$ then the peaks are growing and moving to the left with increasing $n$. This is clear evidence of a phase transition although it is difficult to determine a precise location for the transition. The peaks at smaller values of $y$ are more difficult to interpret. At $a=3$ the peaks largely occur for $y<1$ but the positions may be moving towards $y=1$ with increasing $n$. However, we know rigorously that there is no transition for $y<1$. When $a=5$ the picture is clearer. The peaks occur for values of $y>1$ though their positions do not move smoothly with increasing $n$.

We interpret these results as showing the existence of a mixed phase in this model. There is a transition from the mixed phase to the ballistic phase occurring at a critical value of $y$ that is a function of $a$. There is also a second transition from the adsorbed phase to the mixed phase which must occur at a value of $y \geq 1$, but we cannot be sure exactly where it occurs, or whether its location is a function of $a$.

\section{Series Analysis}

\subsection{Exact enumerations}

Our algorithm for the enumeration of self-avoiding polygons (SAP) on the square lattice is based on the work of Enting [9] who pioneered the use of the finite lattice method. The first terms in the generating function for SAP are calculated using transfer matrix (TM) techniques to count the number of polygons in rectangles $W$ unit cells wide and $L$ cells long. Any polygon spanning such a rectangle has a size of at least $2(W+L)$ edges. Adding contributions from all rectangles of width $W \leq W_{\max }$ and length $W \leq L \leq 2 W_{\max }-W+1$ the number of polygons per vertex of an infinite lattice is obtained correctly up to length $N=4 W_{\max }+2$. Normally one can use the symmetry of the square lattice to restrict the TM calculations to rectangles with $W \leq W_{\max } / 2$ and $L \geq W$ by counting contributions for rectangles with $L>W$ twice. The interactions with the surface breaks

the symmetry and therefore we have to consider all rectangles with $W \leq W_{\max }$. The size of the transfer matrix grows exponentially with $W$ and to partially overcome this hurdle we break the 

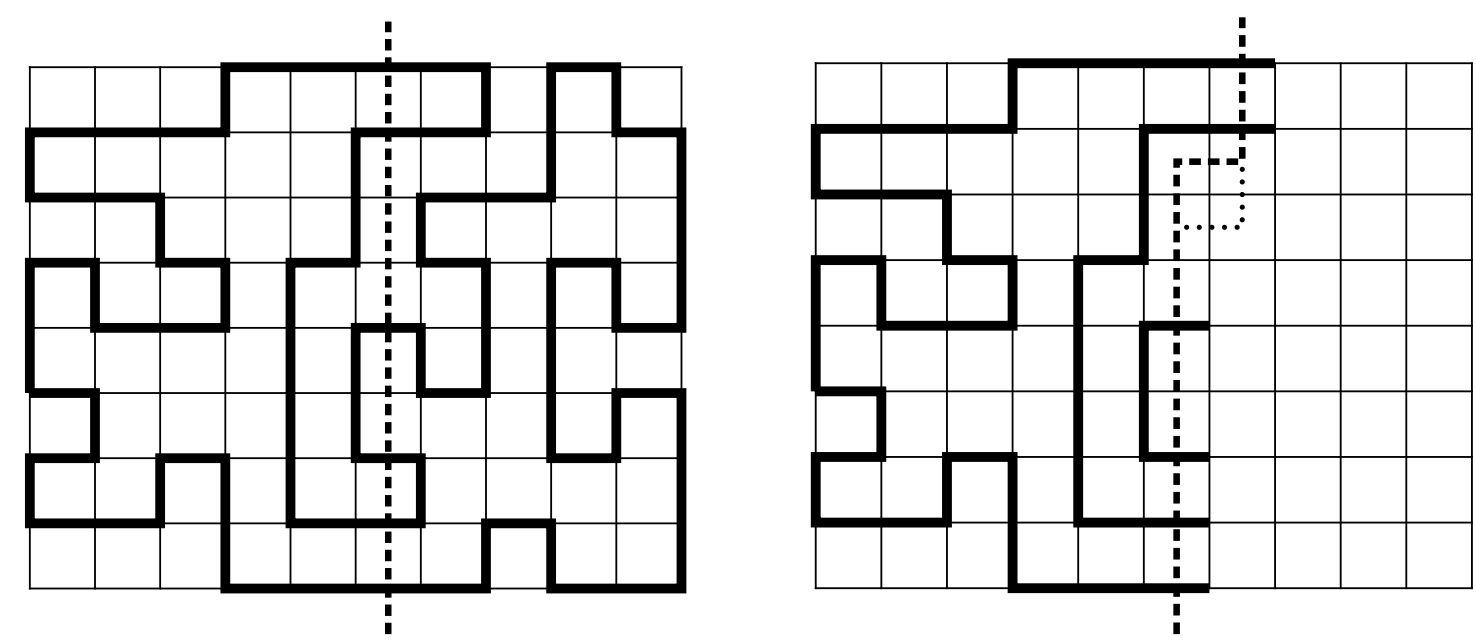

Figure 11. The first panel shows an example of a self-avoiding polygon on a $8 \times 10$ rectangle with a surface on the bottom having 8 vertices in the surface and span 8 . Alternatively we can view it as a SAP on a $10 \times 8$ rectangle with the surface on the left having 7 vertices in the surface and span 10. The cut-line (dashed-line) splits the SAP into a set of arcs to the left (right) of the cut-line. The second panel illustrates how the cut-line is moved in order to build the rectangle vertex by vertex.

TM calculation on the set of rectangles into two sub-sets with $L \geq W$ and $L<W$, respectively. In the calculations for the sub-set with $L \geq W$ the surface is placed on the bottom of the rectangle and for the sub-set with $L<W$ the surface is placed on the left side of the rectangle. The height (or span) $h$ of the SAP is simply $W$ for the first sub-set and $L$ for the second sub-set.

The basic idea of the algorithm is illustrated by the example in Figure 11. Clearly any SAP is topologically equivalent to a circle and when cut by a vertical line (the dashed line in Figure 11) it is broken into several arcs on either side of the cut-line connecting two occupied edges. As the cut-line is moved from left to right we keep track of the ever changing connections (arcs) between occupied edges on the cut-line. Each end of an arc is assigned one of two labels depending on whether it is the lower or upper edge. Any configuration along the cut-line can thus be represented by a set of edge states $\left\{\sigma_{i}\right\}$, where

$$
\sigma_{i}= \begin{cases}0 & \text { empty edge } \\ 1 & \text { lower edge } \\ 2 & \text { upper edge }\end{cases}
$$

Since crossings are not permitted this encoding uniquely describes how the occupied edges are connected. Reading from bottom to top the configuration or signature $S$ along the cut-line of the SAP in Figure 11 is $S=\{111020022\}$ encoding the arcs to the left of the cut-line. The most efficient implementation of the TM algorithm involves moving the cut-line in such a way as to build up the lattice vertex by vertex (see the second panel of Figure 11). The sum over all contributing $\mathrm{SAP}$ is calculated as the cut-line is moved through the lattice. For each signature we maintain a generating function $G_{S}$ for partially completed polygons. Here $G_{S}$ is a truncated polynomial $G_{S}(x, a)$ where $x$ is conjugate to the number of edges in the partially completed polygon and $a$ to the number of visited vertices in the surface. In a TM update each source signature $S$ (before 
the boundary is moved) gives rise to only a few new target signatures $S^{\prime}$ (after the move of the boundary line). In a specific update $k=0,1$ or 2 new edges are occupied and $m=0$ or 1 surface vertices are added (on the bottom or left of the rectangle depending on the sub-set we are dealing with) leading to the update $G_{S^{\prime}}(x, a)=G_{S^{\prime}}(x, a)+x^{k} a^{m} G_{S}(x, a)$. In the case illustrated in Figure 11 the two 'new' edges intersected by the dotted lines are either empty $(k=0)$ or occupied $(k=2)$ if a new arc is inserted.

We calculated the number of SAP up to length $N=100$. The calculation was performed in parallel using up to 32 processors, a maximum of some 70GB of memory and using a total of just under 1000 CPU hours. Details of the implementation and parallelization of our algorithm can be found in $[35,34,12]$.

\subsection{Results}

For SAPs in the bulk, on a bi-partite lattice such as the square, simple-cubic, or indeed hyper-cubic lattice, it is universally believed (though not proved) that

$$
p_{2 n} \sim \operatorname{const} \cdot \mu^{2 n} \cdot n^{\alpha_{b}-3}
$$

where, for the square lattice, $\alpha_{b}=1 / 2$, while for the simple-cubic lattice the best estimate [6] is $\alpha_{b} \approx 0.237209$. However if the polygon sits at a surface and a compressive force (i.e. $y<1$ ) is applied to the top of the polygon, then it has recently been shown by Beaton et al. [4] from probability arguments and particularly assuming SLE predictions that the expected asymptotics now includes a stretched-exponential term. More precisely, for the square lattice,

$$
p_{2 n} \sim \text { const } \cdot \mu^{2 n} \cdot \mu_{1}^{n^{3 / 7}} \cdot n^{-11 / 7},
$$

where both the constant and $\mu_{1}$ are $y$-dependent, and the $y$ dependence of $\mu_{1}$ is also predicted.

In this Section we describe the results from series analysis, chiefly using the method of differential approximants (DAs) [12]. Unfortunately, as discussed in [14], this method has some problems when applied to generating functions whose coefficients have stretched-exponential terms. In particular the estimate of the dominant growth constant $\mu$ produced is much less precise than is usually the case, while the estimates of the critical exponent vary wildly from approximant to approximant. In practical terms, we expect accuracy of 2-4 significant digits in the critical point estimate, while the critical exponent estimate is unobtainable by this method.

We first discuss the $y$-dependence of the free-energy $\lambda_{0}(y)$ when there is no surface interaction (i.e. $a=1$ ), then the $a$-dependence of the free-energy $\kappa_{0}(a)$ when there is no applied force (i.e., $y=1)$ and finally the two variable free-energy $\psi_{0}(a, y)$ when there is both a surface interaction and an applied force.

10.2.1. No surface interaction. $a=1$. If we write

$$
H(x, y)=\sum_{n} P_{2 n}(1, y) x^{n}=\sum_{n} e^{2 \lambda_{0}(y) n+o(n)} x^{n}
$$

where $x$ is the generating variable conjugate to the half-length of the polygon, then $H(x, y)$ will be singular at $x=x_{c}(y)=\exp \left[-2 \lambda_{0}(y)\right]$ and, close to this singularity, $H(x, y)$ is expected to behave as

$$
H(x, y) \sim A\left[x_{c}(y)-x\right]^{\alpha(y)}
$$




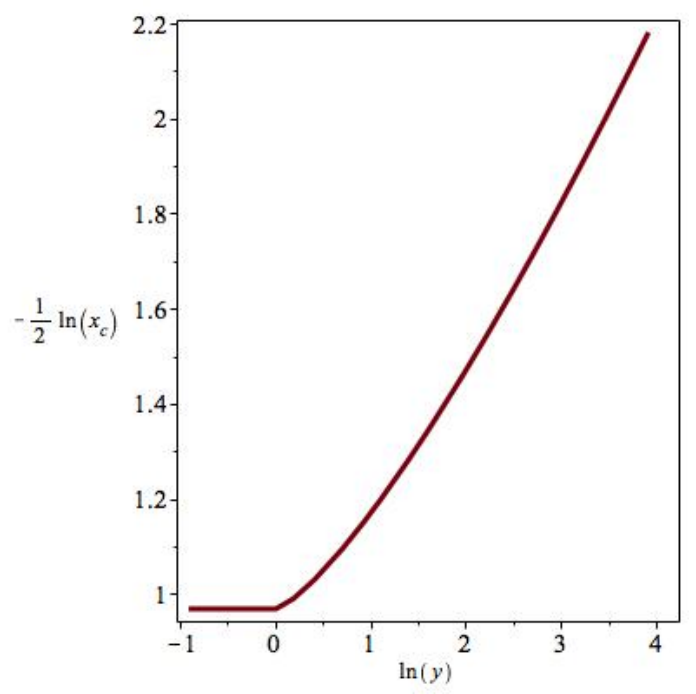

Figure 12. The $y$-dependence of the free-energy $\lambda_{0}(y)$.

where $\alpha(y)$ is a critical exponent whose value depends on $y$.

In the last three columns of Table 1 below we give the results of an analysis of the series $H(x, y)$ for various values of $y$. The resulting estimates of the free-energy $\lambda_{0}(y)=-\frac{1}{2} \log x_{c}$ are plotted in Figure 12. The series were analysed using second and third order differential approximants [12]. At $y=1$ the series is well behaved and has critical point $1 / \mu^{2}$ with exponent $\alpha=3 / 2$, the exponent for self-avoiding polygons, which is unchanged if we consider SAPs attached to a surface (unlike the SAW case).

For $y$ just below 1 the series are quite difficult to analyse, due to the presence of the stretched exponential term. Estimates of $x_{c}$ are moderately close to the known value $1 / \mu^{2}$ in magnitude, but just below $y=1$ they have a small imaginary part. As we lower $y$ further below 1 , we get approximants moderately close to $1 / \mu^{2}$ with very large exponent values, and poor convergence. This is exactly the behaviour discussed in [14] when using the method of differential approximants to analyse series with a stretched-exponential term. The data are consistent with $\mu$ fixed at the bulk (no force) value, and indeed we have proved that in the free region the free-energy stays at the bulk value.

For $y \geq 1.5$ the series are beautifully behaved, the singularity is clearly seen to be a square root, and we can provide 10 digit (or more) accuracy in estimates of the critical point. For $1<y<1.5$ we get the sort of behaviour we expect with a discontinuous change in exponent as we transition from an exponent $3 / 2$ to a square root.

So, in summary, it appears that for $y<1$ we have $x_{c}=1 / \mu^{2}$ and stretched exponential behaviour; for $y=1$ we have $x_{c}=1 / \mu^{2}$ and exponent $\alpha=3 / 2$ and for $y>1$ we have $x_{c}$ monotonically decreasing as $y$ increases, and with a square root singularity.

10.2.2. No applied force. $y=1$. Define the generating function

$$
K(x, a)=\sum_{n} P_{2 n}(a, 1) x^{n}=\sum_{n} e^{2 \kappa_{0}(a) n+o(n)} x^{n} .
$$




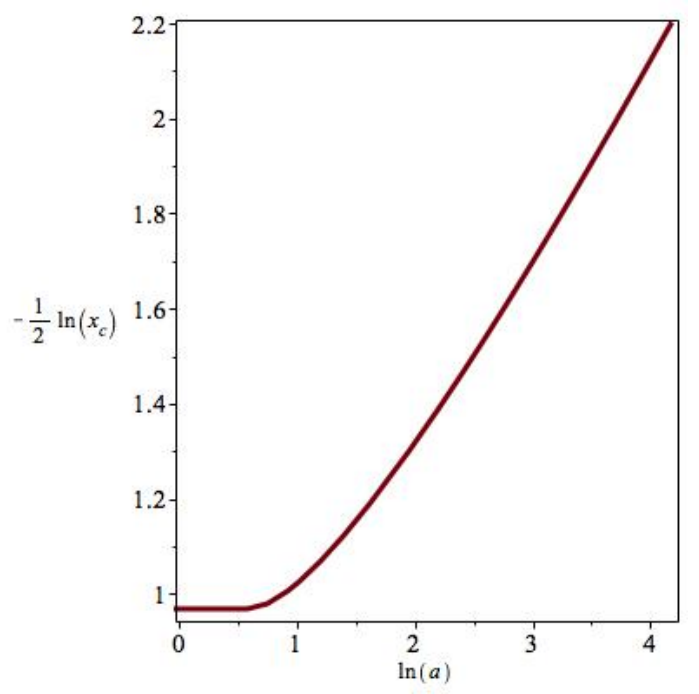

Figure 13. The $a$-dependence of the free-energy $\kappa_{0}(a)$.

$K(x, a)$ will be singular at $x=x_{c}(a)=\exp \left[-2 \kappa_{0}(a)\right]$ and, close to this singularity, $K(x, a)$ should behave as

$$
K(x, a) \sim B\left[x_{c}(a)-x\right]^{\alpha(a)}
$$

where $\alpha(a)$ is a critical exponent whose value depends on $a$.

We have analysed the series $K(x, a)$, corresponding to the "no force" situation, so that $y=1$. Here the differential approximants work well, as there are no stretched-exponential terms and the critical point and exponent are well estimated. The results are shown in the first three columns of Table 1 . If we denote the transition from the free phase to the adsorbed phase by $a_{c}^{o}=\exp \left(-\epsilon / k_{B} T_{c}^{o}\right)$, and denote the corresponding quantity for adsorbed self-avoiding walks by $a_{c}=\exp \left(-\epsilon / k_{B} T_{c}\right)$, we have proved that $a_{c} \leq a_{c}^{o}$. The numerical evidence is extremely strong that equality holds. The best estimate [13] for the SAW case is $a_{c}=1.775615 \pm 0.000005$. From Table 1 , we see that at this value of $a$ the exponent is estimated to be 0.754 which is reasonably close to the conjectured exact value [8] $\alpha^{s p}=3 / 4$, where the superscript refers to the "special" transition that takes place right at the adsorption temperature.

Note that for $a<a_{c}$ the exponent is $3 / 2$. At $a_{c}$ it has changed (presumably discontinuously) to $3 / 4$. We looked at nearby values, and found that at $a=1.774$ the exponent appeared to be 0.763 reflecting a cross-over from 1.5 to 0.75 , while at $a=1.776$ the free-energy has started to change, as the estimate of $x_{c}$ was 0.1436799 , while the exponent was around 0.751 . Thus from the exponent value at $a=1.774$ and the free-energy value at 1.776 we conclude that $a_{c}$ lies between these two values. It therefore seems very likely that $a_{c}^{o}=a_{c}$, and if not, they differ by less than 1 part in a thousand, which seems very unlikely.

So in summary it seems that for $a=a_{c}$ the singularity is characterised by an exponent $3 / 4$, and that this changes discontinuously to a square root for $a>a_{c}$. For $a<a_{c}$ the exponent is, as we would expect, given by $\alpha=3 / 2$. In Figure 13 we give our estimates of the free-energy $\kappa_{0}(a)=-\frac{1}{2} \log x_{c}$ as a function of $\log a$. 


\begin{tabular}{|l|l|l||l|l|l|}
\hline$a(y=1)$ & $x_{c}$ & Exponent & $y(a=1)$ & $x_{c}$ & Exponent \\
\hline 0.5 & 0.143680629 & 1.5000 & 0.4 & 0.147 & 20 \\
1 & 0.143680629 & 1.5000 & 0.7 & 0.1456 & 11 \\
1.775385 & 0.143680629 & 0.754 & 0.9 & $0.1432 \pm 0.0004 i$ & complex \\
2.1 & 0.1406445 & 0.5000 & 1 & 0.143680629 & 1.5000 \\
2.5 & 0.1332540 & 0.5000 & 1.2 & 0.1377 & 0.6 \\
2.75 & 0.1282078 & 0.5000 & 1.5 & 0.12702 & 0.495 \\
3.3 & 0.1175624 & 0.5000 & 2 & 0.1118410 & 0.4998 \\
4 & 0.1058177 & 0.5000 & 2.5 & 0.1000544 & 0.5000 \\
5 & 0.09243473 & 0.5000 & 3 & 0.09075811 & 0.5000 \\
7 & 0.07390853 & 0.5000 & 4 & 0.07703333 & 0.5000 \\
9 & 0.06179279 & 0.5000 & 5 & 0.06733037 & 0.5000 \\
11 & 0.05324182 & 0.5000 & 7 & 0.05436885 & 0.5000 \\
13 & 0.04686712 & 0.5000 & 9 & 0.04598874 & 0.5000 \\
16 & 0.03983702 & 0.5000 & 12 & 0.03768982 & 0.5000 \\
20 & 0.03330407 & 0.5000 & 15 & 0.03213498 & 0.5000 \\
25 & 0.02772693 & 0.5000 & 19 & 0.02701707 & 0.5000 \\
32 & 0.02253994 & 0.5000 & 25 & 0.02196582 & 0.5000 \\
40 & 0.01862508 & 0.5000 & 32 & 0.01814603 & 0.5000 \\
50 & 0.01534307 & 0.5000 & 40 & 0.01521102 & 0.5000 \\
65 & 0.01217273 & 0.5000 & 50 & 0.01270767 & 0.5000 \\
\hline
\end{tabular}

Table 1. SAPs at a surface. Estimates of $x_{c}$ and exponents for $y=1$ and various $a$ values and estimates of $x_{c}$ and exponents for $a=1$ and various $y$ values.

10.2.3. The region $y>1, a<a_{c}$. In this region (and indeed for larger values of $a$, the precise limit depending on the value of $y$ ), we are in the ballistic regime. For fixed $y$, we expect the free-energy to be independent of $a$, until we cross a phase boundary. That this is the case is shown in the first three columns of Table 2, where we show the results for $y=5$. As the value of $a$ increases, the free-energy remains constant until, for a sufficiently large it starts to change with $a$. This constancy is the expected behaviour in the ballistic regime, and it is clear that already at $a=4$ we have transitioned to another regime. We give a second example in Table 3 where we show data for $y=2$. Here we see a transition occuring around $a=2.5$. We examine the nature of this regime below.

10.2.4. The region $y<1$ and $a>a_{c}$. We have seen that for $y<1$ and $a<a_{c}$ we are in the so-called "free" region, the free-energy is constant, but one has stretched-exponential behaviour. For SAWs, when $y<1$ and $a>a_{c}$ one is in the adsorbed regime, and that is the case also for polygons. However in this regime we still observe stretched exponential behaviour, and a freeenergy that depends only on the value of $a$, and agrees with the value given in Table 1 for $y=1$, though our estimates of the free-energy in this regime are less precise than elsewhere because of the stretched exponential behaviour. For this reason, we are also unable to estimate the associated critical exponent in this region. At $y=1$ for $a=a_{c}$ the generating function has a square-root singularity. So this is an adsorbed regime, but with a phase boundary at $y=1$, the nature of 


\begin{tabular}{|l|l|l||l|l|l|}
\hline$a(y=5)$ & $x_{c}$ & Exponent & $y(a=3.45)$ & $x_{c}$ & Exponent \\
\hline 1 & 0.067330372 & 0.5000 & 1.0 & 0.11148643447 & 0.500000 \\
2.5 & 0.06733 & 0.4999 & 1.25 & 0.11 & -1.3 \\
2.75 & 0.06733 & 0.498 & 1.5 & 0.11078 & -0.83 \\
3.0 & 0.067332 & 0.477 & 1.75 & 0.1073 & -0.8 \\
3.25 & 0.06734 & 0.3 & 3.5 & 0.0824875 & -1.1 \\
3.35 & 0.06734 & 0.1 & 4 & 0.076809 & -1.06 \\
3.4 & 0.067343 & -0.1 & 4.5 & 0.07177 & -0.73 \\
3.5 & 0.06733 & -0.452 & 5 & 0.06734 & -0.27 \\
4.0 & 0.066687 & -1.08 & 5.5 & 0.063464 & 0.13 \\
5.0 & 0.0632077 & -1.0000 & 6 & 0.060063 & 0.34 \\
8.0 & 0.05137318 & -1.00000 & 7 & 0.054369 & 0.48 \\
\hline
\end{tabular}

Table 2. SAPs at a surface. Estimates of $x_{c}$ and exponents for $y=5$ and various $a$ values and estimates of $x_{c}$ and exponents for $a=3.45$ and various $y$ values.

which we now examine.

10.2.5. The region $y>1$ and $a>a_{c}$. If one chooses a value of $a>a_{c}$, then as $y$ increases above 1 , we find the free-energy changes monotonically with $y$. An example of this is shown in Table 2, where in the last three columns we show the results of our analysis with $a=3.45$, which is about double the value of $a_{c}$. As $y$ increases the estimates of $x_{c}$ decrease, and though they are not as stable as we might like, the exponent values are initially around -1 , suggesting a simple pole. As $y$ gets sufficiently large, the exponents switch to a square-root. What has happened is that we have gone from a mixed regime where the free-energy depends on both $y$ and $a$, to the ballistic regime.

10.2.6. Phase diagram calculation For SAWs, we were able to locate the phase boundary between the ballistic and the adsorbed region by solving $\kappa(a)=\lambda(y)$ [13]. But for SAPs in the mixed region the free energy, $\psi_{0}$, depends on both $a$ and $y$, so we cannot locate the phase boundary in this way. We know that the phase boundary is on or between the solutions of $\kappa(a)=\lambda(\sqrt{y})$ and $\kappa_{0}(a)=\lambda(\sqrt{y})$. As there is a mixed phase, it follows that the boundary of the ballistic phase cannot coincide with the latter solution, and must be at strictly smaller values of $a$ for each $y>1$.

In fact it seems from our numerical data that the phase boundary does indeed lie on the solution of $\kappa(a)=\lambda(\sqrt{y})$. Consider the data in Table 2. In the first three columns, the data for $y=5$ are given. As $a$ increases, both the free-energy and exponent initially remain essentially constant, as we expect in the ballistic regime. Then between $a=3.25$ and $a=3.5$ the exponent has changed dramatically, from 0.3 to -0.45 , reflecting, we suggest, the transition from the squareroot singularity characteristic of the ballistic regime to the simple-pole behaviour characteristic of the mixed regime. So the phase boundary should lie between these values of $a$. The mid-point is $a=3.375$. From the phase boundary for SAWs, given in [13], we find at $y=\sqrt{5}$ that the point on the phase boundary is at $a=3.379$, remarkably close to our crude estimate.

Now consider the data in the last three columns of Table 2. Here $a=3.45$, and as the value of $y$ increases it is clear that there is a transition from the simple pole behavior for $y \leq 4.5$, toward the square-root behaviour of the ballistic regime when $y>5.5$. So we expect the phase boundary 


\begin{tabular}{|l|l|l|}
\hline$a(y=2)$ & $x_{c}$ & Exponent \\
\hline 1.0 & 0.111841 & 0.4998 \\
1.5 & 0.111841 & 0.4996 \\
2.0 & 0.111841 & 0.49 \\
2.5 & 0.111807 & -0.56 \\
2.6 & 0.11157 & -0.94 \\
3.0 & 0.108584 & -1.01 \\
\hline
\end{tabular}

Table 3. SAPs at a surface. Estimates of $x_{c}$ and exponents for $y=2$ and various $a$ values, showing exponent change as one crosses the phase boundary.

to be at around $y=5$. Again from the phase boundary for SAWs, given in [13], we find at $a=3.45$ that the point on the phase boundary is at $y=2.2593$. Squaring this, we expect the corresponding point on the SAP phase boundary to be at $y \approx 5.10$, again close to our observed transition point.

Our third such calculation involves the data in Table 3. Here $y=2$, and it is clear that the transition from the ballistic to the mixed regime takes place at a value of $a$ around 2.5. Turning to the SAW phase boundary, we find that at $y=\sqrt{2}$, the point on the phase boundary is at $a=2.498$.

So while we cannot identify the phase boundary with the precision that was achieved in the SAW case, all the evidence is consistent with the hypothesis that the phase boundary between the ballistic and mixed phases is given by the solution of $\kappa(a)=\lambda(\sqrt{y})$. Taking this as our working assumption, we show in Figure 14 the phase boundary between the mixed and ballistic phases for SAPs (upper point-plot) and the phase boundary between the ballistic and adsorbed phases for SAWs in the lower point-plot, in the $(\log a, \log y)$-plane.

We can switch to physical variables (force and temperature) using equation (1.4). Without much loss of generality we can set $\epsilon=-1$ and work in units where $k_{B}=1$. The corresponding phase boundary in the force-temperature plane is given in Figure 15. Notice that the force at zero $T$ is 2 and the limiting slope at $T=0$ is zero. For the self-avoiding walk the zero derivative at $T=0$ was predicted in reference [30]. The curve is monotone decreasing as $T$ increases, with no re-entrance.

\section{Conclusions}

When a polymer is adsorbed at a surface it can be desorbed by applying a force normal to the surface to pull the polymer away from the surface. We already have a number of rigorous results available for the self-avoiding walk model of a linear polymer [30, 32, 33]. The behaviour might depend on polymer architecture and we begin to investigate this issue in this paper by considering a self-avoiding polygon model of a ring polymer.

When the dimension is $d \geq 3$ we show that the critical force-temperature curve (i.e. the temperature dependence of the force required to desorb the polygon) can be characterized in terms of the free energy of the adsorbed polygon without a force and the free energy of the polygon subject to a force but not interacting with the surface. Similar results are known for the selfavoiding walk model [30], though we also show that the critical force-temperature curve is different for the polygon case. We are able to determine the phase boundaries in the phase diagram in terms of these free energies. When $d=2$ the situation is more complicated because at most half of the 


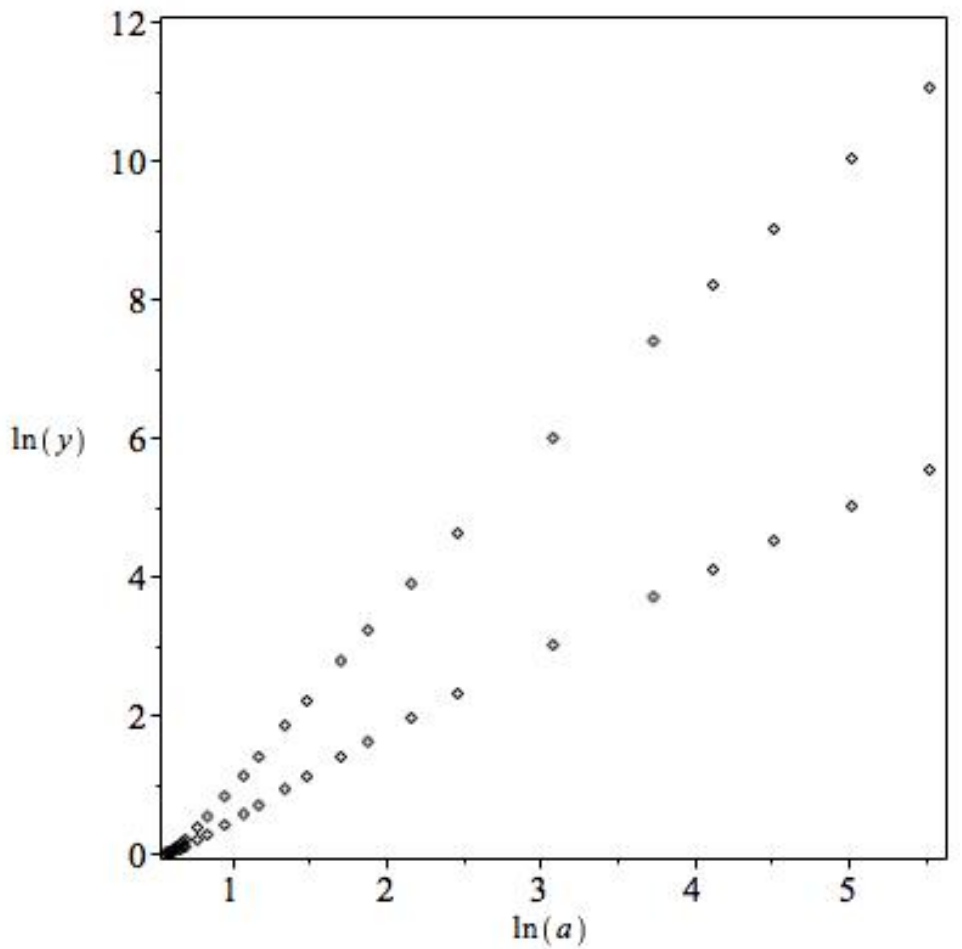

Figure 14. The conjectured phase boundary between the mixed and ballistic phases in the $(\log a, \log y)$-plane for SAPs (upper point-plot) and between the adsorbed and ballistic phases for SAWs (lower point-plot).

vertices of the polygon can be in contact with the surface. For $d=2$ we have bounds on the free energy but our results are less complete.

Our Monte Carlo and exact enumeration results suggest the existence of a mixed phase in two dimensions where the free energy depends on both $a$ and $y$. The results on adsorbing and pulled staircase polygons in reference [2] similarly show a mixed phase which is adsorbed-ballistic in the phase diagram. Mixed phases were also seen in a directed model of copolymer adsorption in reference [21]. The model of adsorbing and pulled staircase polygons is a directed version of our model of two dimensional adsorbing and pulled polygons.

\section{Acknowledgements}

EJJvR and SGW acknowledge support in the form of Discovery Grants from NSERC (Canada). SGW was partially supported by the Leverhulme Trust Research Programme Grant No. RP2013K-009, SPOCK: Scientific Properties of Complex Knots. He would like to acknowledge the hospitality of University of Bristol where some of this research was carried out. AJG and IJ acknowledge support in the form of a Discovery Grant DP140101110 from the ARC (Australia). The computational work of IJ was undertaken with the assistance of resources and services from the National Computational Infrastructure (NCI), which is supported by the Australian Government. 


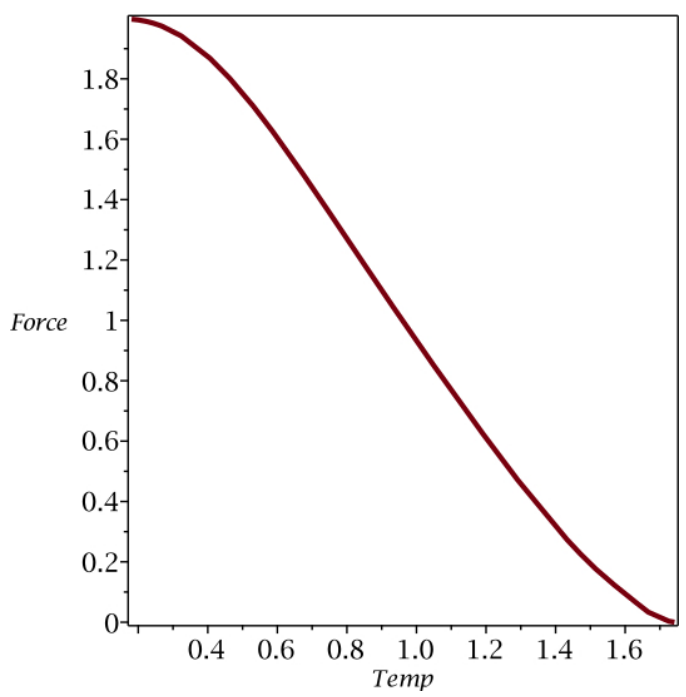

Figure 15. The phase boundary given as a force-temperature diagram. The horizontal axis is the temperature $T=\frac{1}{\log (a)}$, the vertical axis is the force, given by $f=\frac{\log (y)}{\log (a)}$.

\section{References}

[1] Beaton N R 2015 J. Phys. A: Math. Theor. 48 16FT03

[2] Beaton N R 2017 Preprint

[3] Beaton N R, Guttmann A J and Jensen I 2012 J. Phys. A: Math. Theor. 45055208

[4] Beaton N R, Guttmann A J, Jensen I and Lawler G F 2015 J. Phys. A: Math. Theor. 48454001

[5] Berg B and Foesteer D 1981 Phys. Let. B 106 323-326

[6] Clisby N 2010 Phys. Rev. Lett. 10455702

[7] De'Bell K and Lookman T 1993 Rev. Mod. Phys. 65 87-113

[8] Duplantier B 1990 Physics A 163 158-182

[9] Enting I G 1980 J. Phys. A: Math. Gen. 13 3713-3722

[10] Grassberger P 1997 Phys. Rev. E 56 3682-3693

[11] Guim I and Burkhardt T W 1989 J. Phys. A: Math. Gen. 22 1131-1140

[12] Guttmann A J and Jensen I, in Polygons, Polyominoes and Polycubes, ed. A J Guttmann, Lecture Notes in Physics 775 Springer, The Netherlands, (2009)

[13] Guttmann A J, Jensen I and Whittington S G 2014 J. Phys. A: Math. Theor. 47015004

[14] Guttmann A J, 2015 J. Phys. A: Math. Theor. 48045209

[15] Hammersley J M and Morton K W 1954 J. Roy. Stat. Soc. B 16 23-38

[16] Hammersley J M, Torrie G M and Whittington S G 1982 J. Phys. A: Math. Gen. 15 539-571

[17] Hammersley J M and Welsh D J A 1962 Quart. J. Math. Oxford 13 108-110

[18] Hammersley J M and Whittington S G 1985 J. Phys. A: Math. Gen. 18 101-111

[19] Haupt B J, Ennis J and Sevick E M 1999 Langmuir 15 3886-3892

[20] Hegger R and Grassberger P 1994 J. Phys. A: Math. Gen. 27 4069-4081

[21] Iliev G and Janse van Rensburg EJ 2012 J. Stat. Mech: Theo. Expr. P01019

[22] Ioffe D and Velenik Y 2008 Analysis and Stochastics of Growth Processes and Interface Models (ed. Peter Mörters, Roger Moser, Mathew Penrose, Hartmut Schwetlick, and Johannes Zimmer: Oxford University Press Scholarship Online)

[23] Ioffe D and Velenik Y 2010 Braz. J. Prob. Stat. 24 279-299

[24] Janse van Rensburg E J 1998 J. Phys. A: Math. Gen. 31 8295-8306

[25] Janse van Rensburg E J 2010 Cont. Math. 520 127-152

[26] Janse van Rensburg E J 2015 The Statistical Mechanics of Interacting Walks, Polygons, Animals and Vesicles 2ed. Oxford University Press. 
[27] Janse van Rensburg E J, Orlandini E, Tesi M C and Whittington S G 2008 J. Phys. A: Math. Gen. 41015003

[28] Janse van Rensburg E J, Orlandini E, Tesi M C and Whittington S G 2009 J. Stat. Mech. P07014

[29] Janse van Rensburg E J and Rechnitzer A 2004 J. Phys. A: Math. Gen. 37 6875-6898

[30] Janse van Rensburg E J and Whittington S G 2013 J. Phys. A: Math. Theor. 46435003

[31] Janse van Rensburg E J and Whittington S G 2016 J. Phys. A: Math. Theor. 49 11LT01

[32] Janse van Rensburg E J and Whittington S G 2016 J. Phys. A: Math. Theor. 49244001

[33] Janse van Rensburg E J and Whittington S G 2017 J. Phys. A: Math. Theor. 50055001

[34] Jensen I 2003 J. Phys. A: Math. Gen. 36 5731-5745

[35] Jensen I and Guttmann A J 1999 J. Phys. A: Math. Gen. 32 4867-4876

[36] Krawczyk J, Owczarek A L, Prellberg T and Rechnitzer A 2005 J. Stat. Mech. P05008

[37] Mishra P K, Kumar S and Singh Y 2005 Europhys. Letters 69 102-108

[38] Prellberg T and Krawczyk J Phys. Rev. Lett. 92120602

[39] Rechnitzer A and Janse van Rensburg EJ 2008 J. Phys. A: Math. Theo. 41442002

[40] Rychlewski G and Whittington S G 2011 J. Stat. Phys. 45 661-668

[41] Skvortsov A M, Klushin L I, Polotsky A A and Binder K 2012 Phys. Rev. E 85031803

[42] Soteros C E 1992 J. Phys. A: Math. Gen. 25 3153-3173

[43] Soteros C E and Whittington S G 1988 J. Phys. A: Math. Gen. 21 L857-L861

[44] Whittington S G 1975 J. Chem. Phys. 63 779-785

[45] Zhang W and Zhang X 2003 Prog. Polym. Sci. 28 1271-1295 